\title{
METRIC FOUNDATIONS OF CONTINUOUS TRANSFORMATIONS
}

\author{
BY \\ EARL J. MICKLE
}

\section{INTRODUCTION}

In elementary cases the evaluation of a definite integral under a continuous transformation of variables involves the Jacobian of the transformation where the Jacobian is given in terms of derivatives. Furthermore, in elementary cases the transformations involved are usually one-to-one. It is the purpose of this. paper to study the metric foundations of continuous (not necessarily one-to-one) transformations in (complete) metric spaces in order to develop formulas for the transformation of definite integrals. Radó and Reichelderfer $\left.[4]{ }^{1}\right)$ have developed such a theory of metrical and topological properties of continuous transformations in the plane. We shall be concerned with their results on metrical properties and in the next few paragraphs we shall review these results.

Let $\mathcal{D}$ be a bounded, connected open set in the $u v$-plane. A pair of realvalued functions $x(u, v), y(u, v)$, bounded and continuous on $\mathcal{D}$, determines a continuous transformation $T: x=x(u, v), y=y(u, v),(u, v) \in \mathcal{D}$, which in complex notation $z=x+i y, w=u+i v$ can be written in the form $T: z=t(w)$, $w \in \mathcal{D}$. If $E$ is any set in the $w$-plane, then $T(E D)$ will denote the image of $E \mathscr{D}$ in the $z$-plane and $|T(E D)|$ will denote the Lebesgue exterior measure of $T(E D)$. If $\bar{E}$ is any set in the $z$-plane, then $T^{-1}(\bar{E})$ will denote the set of all those points $w \in \mathcal{D}$ whose image is in $\bar{E}$ and this set is termed the inverse of $\bar{E}$ under $T$. The symbol $N(z, T, E)$ will be used to denote the number (possibly equal to $+\infty)$ of distinct points $w$ in the set $E \cdot T^{-1}(z)$.

Let $B$ be a set in the $w$-plane satisfying the conditions (i) $B$ is measurable, and (ii) for every oriented $\left({ }^{2}\right)$ rectangle $R$ such that $\left(^{(}\right) R^{0} C \mathcal{D}$, the set $T\left(R^{0} B\right)$ is a measurable set in the $z$-plane. Such a set $B$ is called a base set. The determination of the base set $B$ for applications depends upon topological properties of $T$ and we shall not be concerned in this paper with such properties except that $B$ is a Borel set.

For each oriented rectangle $R$ such that $R^{0} C \mathcal{D}$ we define $G(R)=\left|T\left(R^{0} B\right)\right|$. A transformation $T$ is called of bounded variation with respect to the base

Presented to the Society, April 26, 1947; received by the editors February 26, 1947.

(1) Numbers in square brackets are used to refer to the bibliography at the end of this paper.

(2) A rectangle $R$ is termed oriented when its sides are parallel to the coordinate axes.

(3) $R^{0}$ denotes the interior of a rectangle $R$. 
set $B$ in $\mathcal{D}$, briefly, B.V.B in $\mathcal{D}$, if there exists a finite constant $L$ such that $G\left(s_{1}\right)+\cdots+G\left(s_{n}\right)<L$ for every finite sequence of nonoverlapping $\left({ }^{4}\right)$, closed, oriented squares $s_{1}, \cdots, s_{n}$ such that $s_{i}^{0} \subset \mathcal{D}$. A transformation $T$ is called absolutely continuous with respect to the base set $B$ in $\mathscr{D}$, briefly, A.C.B in $\Phi$, if for every $\epsilon>0$, there exists an $\eta=\eta(\epsilon)>0$, such that $G\left(s_{1}\right)+\ldots$ $+G\left(s_{n}\right)<\epsilon$ for every finite sequence of nonoverlapping closed, oriented squares $s_{1}, \cdots, s_{n}$ such that $s_{i}^{0} \subset \mathcal{D}$ and $\left|s_{1}\right|+\cdots+\left|s_{n}\right|<\eta$.

Under the measurability assumptions on $B$, the function $N(z, T, \mathcal{D} \cdot B)$ is measurable and $T$ is B.V.B in $\mathcal{D}$ if and only if $N(z, T, \mathcal{D} \cdot B)$ is summable.

Given a point $w \in \mathcal{D}$, let us consider a sequence of oriented closed squares $s_{n}$ such that $w \in s_{n}^{0} \subset \mathcal{D}$ and $\lim \left|s_{n}\right|=0$. If, for every such sequence, the quotients $C\left(s_{n}\right) /\left|s_{n}\right|$ converge to a finite limit (which is then necessarily the same for all such sequences), then this limit is called the derivative of $G(R)$ at $w$ and is denoted by $\dot{G}^{\prime}(w)$. If $T$ is B.V.B in $\mathscr{D}$, then $G^{\prime}(w)$ exists a.e. in $\mathscr{D}$, is measurable and summable in $\mathcal{D}$, and we have, for every open set $O \subset \mathcal{D}$, the inequality

$$
\iint_{o} G^{\prime}(w) \leqq \iint N(z, T, O \cdot B) .
$$

Furthermore, $T$ is A.C. $B$ in $\mathcal{D}$ if and only if the sign of equality holds in the preceding relation for $O=\mathcal{D}$.

Let $H(z)$ be a finite-valued, measurable function in the $z$-plane. Assume that $T$ is A.C. $B$ in $\mathscr{D}$. For every measurable set $E \subset \mathcal{D}$ we then have the transformation formula

$$
\iint_{E} H[t(w)] G^{\prime}(w)=\iint H(z) N(z, T, E \cdot B),
$$

provided only that one of the two integrals involved exists.

We shall show in this paper that results comparable to those of Rado and Reichelderfer [4] can be obtained in complete metric spaces. For this purpose we shall work with analytic sets and the continuous images of analytic sets. Since much of this material is scattered in the literature, for the convenience of the reader we give, in Chapters I and II, the theorems necessary for our theory of continuous transformations. In Chapter III concepts of bounded variation and absolute continuity are introduced and general transformation formulas are given. In Chapter IV the general theory is applied to the above results in the plane.

The essential tool in the development of our theory (see \$2.9) is the decomposition of the set being transformed into a denumerable number of mutually disjoint sets on each of which the transformation is one-to-one plus a set which under our definition of bounded variation plays no role in the

(4) Two squares $s_{1}, s_{2}$ are nonoverlapping if $s_{1}^{0} s_{2}^{0}=0$. 
transformation formulas. The decomposition is such that the transformation formulas can be developed on each of these sets and the general formulas follow from these.

\section{Chapter I. Analytic sets}

1.1. Let 1 denote an abstract space (not assumed to be topologized). If to each finite sequence of positive integers $\phi=\left(n_{1}, n_{2}, \cdots, n_{k}\right)$ there corresponds a set $E_{n_{1} n_{2}} \cdots n_{k}$ (also written $E_{\phi}$ ) of 1 , this denumerable family of sets is called a determining system $\mathfrak{A}$. For a given determining system $\mathfrak{A}$ the set

$$
E=\sum_{\nu} \prod_{k=1}^{\infty} E_{n_{1} n_{2}} \cdots n_{k}
$$

where the products are taken with respect to an infinite sequence of positive integers $\nu=\left(n_{1}, n_{2}, \cdots, n_{k}, \cdots\right)$ and the summation is taken with respect to all infinite sequences of positive integers $\nu$, is called the set derived from the determining system $\mathfrak{A}$ by the operation $\mathcal{A}$. We denote this set $E$ by the symbol

$$
E=\mathcal{A}(\mathfrak{R}), \quad \mathfrak{A}: E_{\phi} .
$$

In the following sections we give some relationships concerning the operation $\mathcal{A}$. In most cases where mere set identities are concerned the proofs are either left as exercises for the reader or suitable references to the literature are given.

1.2. Lemma. Every positive integer $n$ can be written uniquely in the form $n=2^{\alpha-1}(2 \beta-1)$, where $\alpha$ and $\beta$ are positive integers.

1.3. Lemma. For any set $E$ we have that $E=\mathcal{A}(\mathfrak{A}), \mathfrak{A}: E_{\phi}=E$.

1.4. Lemma. If $E=\mathcal{A}(\mathfrak{A}), \mathfrak{A}: E_{\phi}$ and $E^{\prime}=\mathcal{A}\left(\mathfrak{A}^{\prime}\right), \mathfrak{A}^{\prime}: E_{\phi}^{\prime}$, then $E \cdot E^{\prime}$ $=\mathcal{A}\left(\mathfrak{A}^{\prime \prime}\right), \mathfrak{A}^{\prime \prime}: E_{n_{1} n_{2} \cdots n_{k}}^{\prime \prime}=E_{\alpha_{1} \alpha_{2}} \cdots \alpha_{k} \cdot E_{\beta_{1} \beta_{2}}^{\prime} \cdots \beta_{k}$, where $n_{i}=2^{\alpha_{i}-1}\left(2 \beta_{i}-1\right)$ for $i=1,2, \cdots, k$.

1.5. Lemma. If $E^{n}=\mathcal{A}\left(\mathfrak{Q}^{n}\right), \mathfrak{A}^{n}: E_{\phi}^{n}$ for $n=1,2, \cdots$, then

$$
\sum_{n=1}^{\infty} E^{n}=\mathcal{A}(\mathfrak{Y}), \quad \mathfrak{A}: E_{n_{1} n_{2} \cdots n_{k}}=E_{\beta_{1} n_{2} \cdots n_{k}}^{\alpha_{1}},
$$

where $n_{1}=2^{\alpha_{1}-1}\left(2 \beta_{1}-1\right) ;$ and

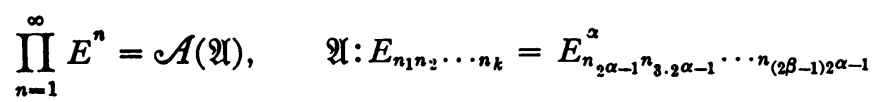

where $k=2^{\alpha-1}(2 \beta-1)$. (See, for example, Hausdorff [2, pp. 90-93].)

1.6. If $\phi=\left(n_{1}, n_{2}, \cdots, n_{k}\right)$ then $(\phi, j)$ will denote the finite sequence of 
positive integers $\left(n_{1}, n_{2}, \cdots, n_{k}, j\right)$ and $(\phi, \nu)$ will denote the infinite sequence of positive integers $\left(n_{1}, n_{2}, \cdots, n_{k}, n_{k+1}, \cdots\right)$ whose first $k$ terms are the same as $\phi$.

1.7. For a set $E=\mathcal{A}(\mathfrak{A}), \mathfrak{A}: E_{\phi}$, we define

$$
Z^{\phi}=\sum_{(\phi, \nu)} \prod_{k=1}^{\infty} E_{n_{1} n_{2} \cdots n_{k}},
$$

where the products are taken with respect to an infinite sequence $(\phi, \nu)$ (see $\S 1.6)$ and the summation is taken with respect to all such infinite sequences $(\phi, \nu)$.

The following relations follow easily from the definition of $Z^{\phi}$.

$$
Z^{\phi} \subset E_{\phi} ; \quad E=\sum_{j=1}^{\infty} Z^{j} ; \quad Z^{\phi}=\sum_{j=1}^{\infty} Z^{(\phi, j)} .
$$

1.8. Lemma. If $E=\mathcal{A}(\mathfrak{A}), \mathfrak{A}: E_{\phi}, E^{\prime}=\mathcal{A}\left(\mathfrak{A}^{\prime}\right), \mathfrak{A}^{\prime}: E_{\phi}^{\prime}$ and for each $\phi$, $Z^{\phi} \subset E_{\phi}^{\prime} \subset E_{\phi}$, then $E^{\prime}=E$.

Proof. Take a point $p \in E$. Then there is an infinite sequence of positive integers $n_{1}, n_{2}, \cdots, n_{k}, \cdots$ such that $p \in E_{n_{1} n_{2} \cdots n_{k}}$ for $k=1,2, \cdots$. Then $p \in Z^{n_{1} n_{2} \cdots n_{k}} \subset E_{n_{1} n_{2}}^{\prime} \cdots n_{k}$ for $k=1,2, \cdots$ and hence $p \in E^{\prime}$. Thus $E \subset E^{\prime}$. Since $E_{\phi}^{\prime} \subset E_{\phi}$ for each $\phi, E^{\prime} \subset E$. Therefore, $E^{\prime}=E$.

1.9. Lemma. If $E=\mathcal{A}(\mathfrak{A}), \mathfrak{A}: E_{\phi}$ then the following inclusion relationship holds (see, for example, Kuratowski [3, p. 6]),

$$
\sum_{j=1}^{\infty} E_{j}-E \subset \sum_{\phi}\left(E_{\phi}-\sum_{j=1}^{\infty} E_{(\phi, j)}\right),
$$

where the denumerable summation on the right is taken with respect to all finite sequences of positive integers $\phi$.

Proof. If $p \in \sum_{j=1}^{\infty} E_{j}-E$ then $p \in E_{n_{1}}$ for some integer $n_{1}$ : If $p \notin E_{n_{1}}$ $-\sum_{j=1}^{\infty} E_{n_{1} j}$ then $p \in E_{n_{1} n_{2}}$ for some integer $n_{2}$. Continuing in this manner we see that if $p$ is not in the right side of the above relationship, there exists an infinite sequence of positive integers $n_{1}, n_{2}, \cdots, n_{k}, \cdots$ such that $p \in E_{n_{1} n_{2} \cdots n_{k}}$ for $k=1,2, \cdots$. Then $p \in E$ which contradicts that $p \in \sum_{j=1}^{\infty} E_{j}$ $-E$. Therefore, if a point $p$ is in the left side of the above relationship it is also in the right side.

1.10. Let $\mathcal{F}$ denote a family of subsets of an abstract space 1 . Let $\Sigma$ denote the operation of adding a denumerable number of sets, $\Pi$ denote the operation of multiplying a denumerable number of sets, $\mathcal{C}$ denote the operation of taking the complement of a set, and $D$ denote the operation of taking the difference of two sets. By a family of sets $K(\mathcal{F}, \Omega)$ we shall mean a family of sets which contains the family of sets $\mathcal{F}$ and is closed under the operations $\Omega$, where $\Omega$ denotes a combination of any of the preceding operations $\Sigma, \Pi, \mathcal{C}, D$. 
For example, $K(\mathcal{F}, \Sigma, \Pi)$ is a family of sets satisfying the conditions: (i) If a set $E \in \mathcal{F}$, then $E \in K(\mathcal{F}, \Sigma, \Pi)$. (ii) If the sets $E_{n} \in K(\mathcal{F}, \Sigma, \Pi)$ for $n=1,2, \cdots$, then the sum of these sets and product of these sets are sets in $K(\mathcal{F}, \Sigma, \Pi)$.

1.11. The product of all families of sets $K(\mathcal{F}, \Omega)$ is again a family of sets $K(\mathcal{F}, \Omega)$. This smallest family of sets $K(\mathcal{F}, \Omega)$ is called the Borel class over $\mathcal{F}$ with respect to the operations $\Omega$ and is denoted by $\mathcal{B}(\mathcal{F}, \Omega)$. When referring to the Borel class over $\mathcal{F}$ without specifying the operations we shall mean the usual class $\mathbb{B}(\mathcal{F}, \Sigma, \mathcal{C})$. If $M$ is a metric space and $\mathcal{F}$ is the class of closed (or open) subsets of $M$, we shall refer to $\mathbb{B}(\mathcal{F}, \Sigma, \mathcal{C})$ as the class of Borel subsets of $M$.

1.12. If $\mathcal{F}$ contains the empty set, then a family of sets $K(\mathcal{F}, \Sigma, \mathcal{C})$ is called a completely additive class of sets. A completely additive class of sets is also a family of sets $K(\mathcal{F}, \Sigma, \mathcal{C}, \Pi, D)$ (see, for example, Saks [5, p. 7]).

1.13. Lemma. If $M$ is a metric space and $\mathcal{F}$ is the class of closed subsets of $M$, then $\mathcal{B}(\mathcal{F}, \Sigma, \Pi) \equiv \mathbb{B}(\mathcal{F}, \Sigma, \mathcal{C})$.

Proof. From $\S 1.12, \mathbb{B}(\mathcal{F}, \Sigma, \mathcal{C})$ is a family of sets $K(\mathcal{F}, \Sigma, \mathcal{C}, \Pi, D)$. Hence $\mathbb{B}(\mathcal{F}, \Sigma, \mathcal{C}) \supset \mathbb{B}(\mathcal{F}, \Sigma, \Pi)$.

Let $\mathcal{B}^{\prime}$ be the chass of sets $E$ of $\mathcal{B}(\mathcal{F}, \Sigma, \Pi)$ satisfying that if $E \in \mathcal{B}(\mathcal{F}, \Sigma, \Pi)$ then $\mathcal{C} E \in \mathcal{B}(\mathcal{F}, \Sigma, \Pi)$. Let $F$ be a closed set and let $F_{n}$ be the set of points of $M$ that are a distance greater than or equal to $1 / n$ from $F$. Then $F_{n}$ is a closed set and $\mathcal{C} F=F_{1}+\cdots+F_{n}+\cdots$ is in $\mathcal{B}(\mathcal{F}, \Sigma, \Pi)$. Hence $F \in \mathcal{B}^{\prime}$. Let $E_{n}, n=1,2, \cdots$ be a sequence of sets in $\mathcal{B}^{\prime}$. Then $E=E_{1}+\cdots+E_{n}+\cdots$ is in $\mathcal{B}(\mathcal{F}, \Sigma, \Pi)$ and $\mathcal{C} E=\prod_{n=1}^{\infty} \mathcal{C} E_{n}$ is a set in $\mathcal{B}(\mathcal{F}, \Sigma, \Pi)$. Hence $E$ is in $\mathcal{B}^{\prime}$. Thus $\mathcal{B}^{\prime}$ is a family of sets $K(\mathcal{F}, \Sigma, \mathcal{C})$. Thus $\mathcal{B}(\mathcal{F}, \Sigma, \Pi) \supset \mathcal{B}^{\prime} \supset \mathcal{B}(\mathcal{F}, \Sigma, \mathcal{C})$ $\supset \mathbb{B}(\mathcal{F}, \Sigma, \Pi)$. Therefore $\mathbb{B}(\mathcal{F}, \Sigma, \Pi) \equiv \mathcal{B}(\mathcal{F}, \Sigma, \mathcal{C})$.

1.14. The Souslin class over a family of sets $\mathcal{F}$, which we denote by $\mathcal{S}(\mathcal{F})$, is the family of sets derived by the operation $\mathcal{A}$ on all possible determining systems of $\mathcal{F}$, that is, a set $E \in \mathcal{S}(\mathcal{F})$ if $E=\mathscr{A}(\mathfrak{A}), \mathfrak{A}: E_{\phi} \in \mathcal{F}$.

From $\S 1.3$ and $\S 1.5$ it follows that $\delta(\mathcal{F})$ is a family of sets $K(\mathcal{F}, \Sigma, \Pi)$ and hence $S(\mathcal{F}) \supset \mathbb{B}(\mathcal{F}, \Sigma, \Pi)$.

1.15. Let $M$ be a metric space. Let $\mathcal{F}$ be the class of all closed subsets of $M$. Sets in $\mathcal{S}(\mathcal{F})$ (see $\$ 1.14$ ) are called analytic sets. From $\$ 1.13, \S 1.14$ it follows that every Borel subset of $M$ is an analytic set.

1.16. Let $\mathcal{X}$ be a completely additive class of sets (see $\$ 1.12$ ). A set function $\Phi(E)$, defined (possibly equal to $+\infty$ ) and non-negative for sets $E \in \mathcal{X}$, is called a measure on $\mathcal{X}$ if $\Phi\left(E_{1}+\cdots+E_{n}+\cdots\right)=\Phi\left(E_{1}\right)+\cdots$ $+\Phi\left(E_{n}\right)+\cdots$ for every denumerable sequence of sets $E_{n} \in \mathcal{X}$ such that $E_{\xi} E_{j}=0, i \neq j$.

1.17. Let $M$ be a metric space. A set function $\Phi(E)$, defined (possibly equal to $+\infty)$ and non-negative for every subset $E$ of $M$, is called a Caratheodory outer measure if (i) $\Phi(E)=0$ if $E$ is the empty set, (ii) $\Phi\left(E_{1}\right)$ $\leqq \Phi\left(E_{2}\right)$ whenever $E_{1} \subset E_{2}$, (iii) $\Phi\left(E_{1}+\cdots+E_{n}+\cdots\right) \leqq \Phi\left(E_{1}\right)+\cdots$ 
$+\Phi\left(E_{n}\right)+\cdots$ for every denumerable sequence of sets $E_{n}$, (iv) $\Phi\left(E_{1}+E_{2}\right)$ $=\Phi\left(E_{1}\right)+\Phi\left(E_{2}\right)$ whenever the distance between $E_{1}$ and $E_{2}$ is positive.

A set $E$ is called measurable with respect to a given Carathéodory outer measure $\Phi$ if for every subset $Q$ of $M, \Phi(Q)=\Phi(Q E)+\Phi(Q \cdot \mathcal{C} E)$. It follows immediately that every set $E$ for which $\Phi(E)=0$ is measurable. We have (see, for example, Saks [5, pp. 43-52]):

THEOREM. Let $\Phi(E)$ be a Carathéodory outer measure for sets $E$ in a metric space $M$. Then the class $\mathcal{X}$ of measurable sets is a completely additive class of sets (see \$1.12) containing the class of Borel subsets of $M$ and $\Phi(E)$ is a measure on $\mathcal{X}($ see $\$ 1.16)$.

If for each set $E$, there is a measurable set $H \supset E$ such that $\Phi(E)=\Phi(H)$, then $\Phi(E)$ is called a regular Carathéodory outer measure. The set $H$ is called a measurable hull of $E$.

If $\Phi(M)$ is finite we shall call $\Phi(E)$ a finite Carathéodory outer measure.

LemMa. If $\Phi(E)$ is a finite Carathéodory outer measure and $H$ is a measurable hull of $E$, then (i) for every measurable set $H_{1} \subset H-E$, we have $\Phi\left(H_{1}\right)=0$, and (ii) for every measurable set $\mathrm{H}_{2} \supset E, \mathrm{HH}_{2}$ is also a measurable hull of $E$.

Proof. To prove (i) let $H_{1}$ be a measurable subset of $H-E$. Then $\Phi(E)$ $=\Phi(H)=\Phi\left(H-H_{1}\right)+\Phi\left(H_{1}\right) \geqq \Phi(E)+\Phi\left(H_{1}\right)$. Thus (i) follows.

To prove (ii) let $\mathrm{H}_{2}$ be a measurable set containing $E$. Then $E \subset H H_{2}$ and $\Phi(E)=\Phi(H) \geqq \Phi\left(H H_{2}\right) \geqq \Phi(E)$. Hence $H_{2}$ is a measurable hull of $E$.

1.18. THEOREM. Let $\Phi(E)$ be a finite regular Carathéodory outer measure for sets $E$ in a metric space $M$ and let $\mathcal{X}$ be the class of sets measurable with respect to $\Phi$ (see $\$ 1.17)$. Then $\mathcal{X}$ contains the class of analytic sets (see $\$ 1.15)$.

Proof. This theorem is true even if $\Phi$ is a general Caratheodory outer measure (see, for example, Saks [5, pp. 47-50]) but we shall give a proof for the case where $\Phi$ is finite and regular (see Kuratowski $[3$, p. 58]). Since $\mathcal{X}$ contains the closed subsets of $M$ the theorem will be proved by showing that $\mathcal{S}(\mathscr{H}) \equiv \mathcal{X}$ (see $\S 1.14)$. Let $E=\mathcal{A}(\mathfrak{A}), \mathfrak{A}: E_{\phi} \in \mathcal{X}$. Let $H_{\phi}$ be a measurable hull of the set $Z^{\phi}$ defined in $\$ 1.7$. By the lemma in $\$ 1.17, E_{\phi}^{\prime}=H_{\phi} E_{\phi}$ is also a measurable hull of $Z^{\phi}$ and satisfies the conditions $Z^{\phi} \subset E_{\phi}^{\prime} \subset E_{\phi}$. Then by $\S 1.8, E=\mathcal{A}\left(\mathfrak{H}^{\prime}\right), \mathfrak{A}^{\prime}: E_{\phi}^{\prime} \in \mathcal{X}$. For each $\phi$ (see $\left.\S 1.7\right)$

$$
E_{\phi}^{\prime}-\sum_{j=1}^{\infty} E_{(\phi, j)}^{\prime} \subset E_{\phi}^{\prime}-\sum_{j=1}^{\infty} Z^{(\phi, j)}=E_{\phi}^{\prime}-Z^{\phi} .
$$

Since $E_{\phi}^{\prime}$ is a measurable hull of $Z^{\phi}$, by the lemma in $\$ 1.17$, for each $\phi$

$$
\Phi\left(E_{\phi}^{\prime}-\sum_{j=1}^{\infty} E_{(\phi, j)}^{\prime}\right)=0 .
$$


By the inclusion relationship of $\S 1.9$

$$
\sum_{j=1}^{\infty} E_{i}^{\prime}-E \subset \sum_{\phi}\left(E_{\phi}^{\prime}-\sum_{j=1}^{\infty} E_{(\phi, j)}^{\prime}\right) .
$$

Since the right side of this last relation is a denumerable summation of sets of $\Phi$ measure zero, the sum of these sets is a set of $\Phi$ measure zero. Hence the set on the left side is of $\Phi$ measure zero. Since each set $E_{j}^{\prime} \in \mathcal{X}$ and the sum of these sets contains $E$, it follows that $E \in \mathcal{X}$. Thus $S(\mathscr{X}) \subset \mathcal{X}$. From $\S 1.3$, $\mathscr{X} \subset \mathcal{S}(\mathscr{X})$. Therefore, $S(\mathscr{X}) \equiv \mathcal{X}$ and the theorem follows.

1.19. If $\Phi(E)$ is a finite Carathéodory outer measure and if for every set $E, \Phi(E)=$ g.l.b. $\Phi(O)$, where the greatest lower bound is taken with respect to all open sets $O$ which contain $E$, we shall say that $\Phi(E)$ is a finite Carathéodory outer measure which is regular with respect to open sets.

If $\Phi(E)$ is a finite Carathéodory outer measure which is regular with respect to open sets then it is easily shown that for every measurable set $E$, there exists Borel sets $B_{1}, B_{2}$ such that $B_{1} \subset E C B_{2}$ and $\Phi\left(B_{1}\right)=\Phi(E)=\Phi\left(B_{2}\right)$. In fact the sets $B_{1}$ and $B_{2}$ can be taken respectively as the sum of a denumerable number of closed sets and the product of a denumerable number of open sets.

\section{Chapter II. Continuous transformations in metric SPACES}

2.1. Let $M, M^{*}$ be two metric spaces. A transformation $T: p^{*}=t(p)$, $p \in A \subset M, p^{*} \in A^{*} \subset M^{*}$ is called a single-valued, continuous transformation from a set $A \subset M$ onto a set $A^{*} \subset M^{*}$ if (i) for each point $p \in A$ there is one and only one point $p^{*} \in A^{*}$ for which $p^{*}=t(p)$, (ii) for each point $p^{*} \in A^{*}$ there is at least one point $p \in A$ for which $p^{*}=t(p)$, (iii) for each sequence of points $p_{n}$ such that $p_{n} \in A, p_{n} \rightarrow p_{0} \in A$, the sequence $t\left(p_{n}\right) \rightarrow t\left(p_{0}\right)$.

For each set $E \subset M, T(E A)$ will denote the set of those points $p^{*}$ of $A^{*}$ for which there is a point $p \in E A$ such that $p^{*}=t(p)$. For each set $E^{*} \subset M^{*}$, $T^{-1}\left(E^{*}\right)$ (called the inverse of the set $E^{*}$ under $T$ ) will denote the set of those points $p$ of $A$ for which $t(p) \in E^{*}$.

We shall use the symbol $N\left(p^{*}, T, E\right)$ to denote the number (possibly equal to $+\infty)$ of distinct points in the set $E A \cdot T^{-1}\left(p^{*}\right)$.

2.2. A transformation $T$ is called univalent on a set $E$ if $T^{-1}\left(p^{*}\right) \cdot E$ is a single point for each point $p^{*} \in T(E)$.

2.3. A metric space $M$ is called complete if every Cauchy sequence of point in $M$ has a limit point in $M$. A metric space $M$ is called separable if there is a dense denumerable subset of $M$.

2.4. Let $M$ be a separable metric space containing a dense denumerable subset of points $x_{1}, x_{2}, \cdots, x_{n}, \cdots$. Let $S_{n_{1} n_{2} \cdots n_{k}}$ denote the closed sphere of radius $1 / k$ and center at $x_{n_{k}}$. From the definition of the operation $\mathcal{A}$ (see \$1.1) it follows that 


$$
M=\mathcal{A}(\mathfrak{R}), \quad \mathfrak{A}: S_{n_{1} n_{2} \cdots n_{k}} .
$$

Let $d\left(p_{1}, p_{2}\right)$ denote the distance between two points $p_{1}, p_{2}$ of $M$ and let $d(E)$ denote the diameter of a set $E \subset M$ (the diameter of a set $E$ is the least upper bound of $d\left(p_{1}, p_{2}\right)$ for points $p_{1}, p_{2}$ in $\left.E\right)$.

Lemma. If $E=\mathcal{A}(\mathfrak{A}), \mathfrak{A}: E_{\phi}$ where the sets $E_{\phi}$ are closed sets, then there exists a determining system $\mathfrak{A}^{\prime}$ such that $E=\mathcal{A}\left(\mathfrak{A}^{\prime}\right), \mathfrak{A}^{\prime}: E_{\phi}^{\prime}$, the sets $E_{\phi}^{\prime}$ are closed sets, and $d\left(E_{n_{1} n_{2}}^{\prime} \cdots n_{k}\right) \rightarrow 0$ for $k \rightarrow \infty$.

Proof. By the preceding paragraph and $\S 1.4, E=E \cdot M=\mathcal{A}\left(\mathfrak{A}^{\prime}\right)$, $\mathfrak{A}^{\prime}: E_{n_{1} n_{2}}^{\prime} \cdots n_{k}=E_{\alpha_{1} \alpha_{2} \cdots \alpha_{k}} \cdot S_{\beta_{1} \beta_{2}} \cdots \beta_{k}$, where $n_{i}=2^{\alpha_{i}-1}\left(2 \beta_{i}-1\right), i=1,2, \cdots, k$. The sets $E_{\phi}^{\prime}$ are closed and $d\left(E_{n_{1} n_{2} \cdots n_{k}}^{\prime}\right) \rightarrow 0$ for $k \rightarrow \infty$, since $d\left(E_{n_{1} n_{2} \cdots n_{k}}^{\prime}\right)$ $\leqq d\left(S_{\beta_{1} \beta_{2} \ldots \beta_{k}}\right) \leqq 2 / k$.

2.5. A determining system $\mathfrak{A}$ such that $E_{n_{1} n_{2} \cdots n_{k}} \supset E_{n_{1} n_{2} \cdots n_{k} n_{k+1}}$ is called a regular determining system.

Lemma. If $E=\mathcal{A}(\mathfrak{A}), \mathfrak{A}: E_{\phi}$, where the sets $E_{\phi}$ are closed sets, then there exists a regular determining system $\mathfrak{A}^{\prime}$ such that $E=\mathcal{A}\left(\mathfrak{H}^{\prime}\right), \mathfrak{A}^{\prime}: E_{\phi}^{\prime}$ and the sets $E_{\phi}^{\prime}$ are closed sets.

Proof. The proof follows immediately by setting $E_{n_{1} n_{2} \cdots n_{k}}^{\prime}=E_{n_{1}} \cdot E_{n_{1} n_{2}}$ $\cdots E_{n_{1} n_{2} \cdots n_{k}}$.

2.6. Theorem. If $T: p^{*}=t(p), p \in A \subset M, p^{*} \in A^{*} \subset M^{*}$ is a single-valued continuous transformation from an analytic set $A$ in a complete and separable metric space $M$ onto a set $A^{*}$ in a metric space $M^{*}$, then $A^{*}$ is an analytic set.

Proof. (See, for example, Hausdorff [2, p. 209].) Since $A$ is an analytic set, by $\S \S 2.5$ and 2.4 there is a regular determining system of closed sets $\mathfrak{A}$ such that $A=\mathcal{A}(\mathfrak{A}), \mathfrak{A}: A_{\phi}$ and $d\left(A_{n_{1} n_{2}} \ldots n_{k}\right) \rightarrow 0$ for $k \rightarrow \infty$. We shall now show that $A^{*}=B^{*}$ where

$$
B^{*}=\mathcal{A}\left(\mathfrak{B}^{*}\right), \quad \mathfrak{B}^{*}: B_{n_{1} n_{2}}^{*} \cdots n_{k}=\operatorname{closure}\left({ }^{(5)}\right) \text { of } T\left(A \cdot A_{n_{1} n_{2} \cdots n_{k}}\right) .
$$

(i) Take a point $p_{0}^{*} \in A^{*}$. Then there is a point $p_{0} \in A$ such that $p_{0}^{*}=t\left(p_{0}\right)$ and an infinite sequence of positive integers $\nu=\left(n_{1}, n_{2}, \cdots, n_{k}, \cdots\right)$ such that $p_{0} \in A_{n_{1} n_{2} \cdots n_{k}}$ for $k=1,2, \cdots$. Hence $p_{0}^{*} \in B_{n_{1} n_{2} \cdots n_{k}}^{*}$ for $k=1,2, \cdots$ and $p_{0}^{*} \in B^{*}$. Therefore, $A^{*} \subset B^{*}$.

(ii) Take a point $p_{0}^{*} \in B^{*}$. Then there is an infinite sequence of integers $\nu=\left(n_{1}, n_{2}, \cdots, n_{k}, \cdots\right)$ such that $p_{0}^{*} \in B_{n_{1} n_{2} \cdots n_{k}}^{*}$ for $k=1,2, \cdots$. Then for each integer $k$ there exists points $p_{k}^{*} \in T\left(A \cdot A_{n_{1} n_{2} \cdots n_{k}}\right), p_{k}=A \cdot A_{n_{1} n_{2}} \cdots n_{k}$ such that $d\left(p_{k}^{*}, p_{0}^{*}\right)<1 / k$ and $p_{k}^{*}=t\left(p_{k}\right)$. Since $d\left(A_{n_{1} n_{2} \cdots n_{k}}\right) \rightarrow 0$ for $k \rightarrow \infty, p_{k}$ is a Cauchy sequence of points and (since $M$ is a complete space) converges to a point $p_{0} \in M$. Since $p_{k} \in A_{n_{1} n_{2} \cdots n_{j}}$ for every $j \leqq k$ ( $\mathfrak{A}$ is a regular determin-

(b) The closure of a set $E$ is the set $E$ plus all its limit points. Hence, the closure of a set is a closed set. 
ing system) and since the sets $A_{n_{1} n_{2}} \cdots n_{k}$ are closed, the point $p_{0} \in A_{n_{1} n_{2} \cdots n_{k}}$ for $k=1,2, \cdots$. Thus $p_{0} \in A$ and $t\left(p_{0}\right) \in A^{*}$. Since $T$ is continuous on $A$, the sequence $p_{k}^{*}=t\left(p_{k}\right)$ converges to $t\left(p_{0}\right)$ for $k \rightarrow \infty$. On the other hand, since $d\left(p_{k}^{*}, p_{0}^{*}\right)<1 / k$, the sequence $p_{k}^{*} \rightarrow p_{0}^{*}$ for $k \rightarrow \infty$. Therefore. $p_{0}^{*}=t\left(p_{0}\right)$ and $p_{0}^{*} \in A^{*}$. Hence $A^{*} \supset B^{*}$.

From (i) and (ii), $A^{*}=B^{*}$. Hence $A^{*}$ is an analytic set.

2.7. TheOREM. Under the same assumptions as $\$ 2.6$, if $B^{*}$ is an analytic set in $A^{*}$, then $T^{-1}\left(B^{*}\right)$ is an analytic set.

Proof. Since $B^{*}$ is an analytic set there exists a regular determining system $\mathfrak{B}^{*}$ of closed sets $B_{\phi}^{*}$ such that $B^{*}=\mathcal{A}\left(\mathfrak{B}^{*}\right), \mathfrak{B}^{*}: B_{\phi}^{*}$. We shall show that $T^{-1}\left(B^{*}\right)=A B$, where

$$
B=\mathcal{A}(\mathfrak{B}), \quad \mathfrak{B}: B_{n_{1} n_{2} \cdots n_{k}}=\text { closure of } T^{-1}\left(B^{*} \cdot B_{n_{1} n_{2} \cdots n_{k}}^{*}\right) .
$$

(i) Let $p_{0} \in T^{-1}\left(B^{*}\right)$. Then $t\left(p_{0}\right)=p_{0}^{*} \in B^{*}$ and we have an infinite sequence of integers $\nu=\left(n_{1}, n_{2}, \cdots, n_{k}, \cdots\right)$ such that $p_{0}^{*} \in B_{n_{1} n_{2} \cdots n_{k}}$ for $k=1,2, \cdots$. Hence $p_{0} \in T^{-1}\left(p_{0}^{*}\right) \subset B_{n_{1} n_{2} \cdots n_{k}}$ for $k=1,2, \cdots$. Thus $p_{0} \in B$. Therefore, $T^{-1}\left(B^{*}\right) \subset A B$.

(ii) Let $p_{0} \in A B$. Then we have an infinite sequence of integers $\nu=\left(n_{1}, n_{2}, \cdots, n_{k}, \cdots\right)$ such that $p_{0} \in B_{n_{1} n_{2} \cdots n_{k}}$ for $k=1,2, \cdots$. For each integer $k$ we have a point $p_{k}$ such that $p_{k} \subset T^{-1}\left(B^{*} \cdot B_{n_{1} n_{2}}^{*} \cdots n_{k}\right)$ and $d\left(p_{k}, p_{0}\right)$ $<1 / k$. Thus $t\left(p_{k}\right) \in B_{n_{1} n_{2}}^{*} \cdots_{n_{j}}$ for every $j \leqq k$ (since $\mathfrak{B}^{*}$. is a regular determining system). Since $p_{k} \rightarrow p_{0}$ and each $B_{\phi}^{*}$ is closed, $t\left(p_{0}\right) \in B_{n_{1} n_{2} \cdots n_{k}}^{*}$ for $k=1,2, \cdots$. Thus $t\left(p_{0}\right) \in B^{*}$ and $p_{0} \in T^{-1}\left(B^{*}\right)$. Therefore, $A B \subset T^{-1}\left(B^{*}\right)$.

From (i) and (ii), $A B=T^{-1}\left(B^{*}\right)$ and $T^{-1}\left(B^{*}\right)$ is an analytic set.

2.8. THEOREM. Under the same assumptions as \$2.6, if for each positive integer $k, A_{k}^{*}$ denotes the set of points $p^{*}$ such that $N\left(p^{*}, T, A\right) \geqq k$ (see $\left.\$ 2.1\right)$ then $A_{k}{ }^{*}$ is an analytic set.

Proof. (See, for example, Hahn [1, pp. 361-362].) Let all open spheres of rational radius and centers at a dense denumerable subset of $M$ be arranged in a sequence $S_{1}, S_{2}, \cdots$. Set

$$
B_{k}^{*}=\sum T\left(A S_{n_{1}}\right) \cdot T\left(A S_{n_{2}}\right) \cdots T\left(A S_{n_{k}}\right) .
$$

where the summation is-taken with respect to all sets of $k$ nonoverlapping open spheres $S_{n_{1}}, S_{n_{2}}, \cdots, S_{n_{k}}$. We shall show that $B_{k}{ }^{*}=A_{k}{ }^{*}$.

(i) Take a point $p_{0}^{*} \in A_{k}^{*}$. Then $p_{0}{ }^{*}$ has $k$ models in $A$ which can be enclosed in $k$ nonoverlapping spheres $S_{n_{1}}, S_{n_{2}}, \cdots, S_{n_{k}}$ each one of which contains one of the $k$ models. Thus $p_{0}^{*} \in T\left(A S_{n_{1}}\right) \cdot T\left(A S_{n_{2}}\right) \cdots T\left(A S_{n_{k}}\right) \subset B_{k}^{*}$. Therefore, $A_{k}^{*} \subset B_{k}^{*}$.

(ii) Take a point $p_{0}^{*} \in B_{k}^{*}$. Then $p_{0}^{*}$ is in one of the terms $T\left(A S_{n_{1}}\right)$ - $T\left(A S_{n_{2}}\right) \cdots T\left(A S_{n_{k}}\right)$ of the summation defining $B_{k}^{*}$ and hence has a model 
in each one of these $k$ nonoverlapping open spheres $S_{n_{1}}, S_{n_{2}}, \cdots, S_{n_{k}}$. Hence $p_{0}^{*} \in A_{k}^{*}$ and $B_{k}^{*} \subset A_{k}^{*}$.

From (i) and (ii) it follows that $A_{k}^{*}=B_{k}^{*}$. By $\$ 2.6, T\left(A S_{n}\right)$ is an analytic set and hence, by $\$ 1.5$, each term of the denumerable summation defining $B_{k}{ }^{*}$ is an analytic set. Since the summation is a denumerable summation, by $\S 1.5, B_{k}^{*}$ is an analytic set.

Corollary. Under the same assumptions as $\$ 2.6$, the set $A_{\infty}^{*}$ of those points $p^{*}$ for which $N\left(p^{*}, T, A\right)=+\infty$ and the set $A_{\infty}=T^{-1}\left(A_{\infty}^{*}\right)$ are analytic sets.

Proof. By the preceding theorem and $\S 1.5, A_{\infty}^{*}=A_{1}^{*} A_{2}{ }^{*} \cdots A_{n}^{*} \cdots$ is an analytic set. By $\S 2.7, A_{\infty}$ is also an analytic set.

2.9. Theorem. If $T: p^{*}=t(p), p \in A \subset M, p^{*} \in A^{*} \subset M^{*}$ is a single-valued, continuous transformation from an analytic set $A$ in a complete and separable metric space $M$ onto a set $A^{*}$ in a metric space $M^{*}$, then there exists a denumerable number of. sets $X_{1}, \cdots, X_{n}, \cdots$ satisfying the following conditions: (1) $A=A_{\infty}+X_{1}+\cdots+X_{n}+\cdots$ (2) $A_{\infty} \cdot\left(X_{1}+\cdots+X_{n}+\cdots\right)=0$. (3) $X_{i} X_{j}=0, i \neq j$. (4) $T$ is univalent (see §2.2) on $X_{n}, n=1,2, \cdots$ (5) $X_{n}$ and $T\left(X_{n}\right)$ are the difference of two analytic sets for $n=1,2, \ldots$

Proof. Let all open spheres with rational radii and centers at the points of a dense denumerable subset of $M$ be arranged in a sequence $S_{1}, \cdots$, $S_{n}, \cdots$. Set $B_{1}=A, E_{1}=B_{1} S_{1}+A_{\infty}$,

$$
Y_{1}=\underset{p}{E}\left\{p \in E_{1}, N\left[p^{*}=t(p), T, E_{1}\right]>1\right\},
$$

$X_{1}=E_{1}-Y_{1}$. By applying the results of $\S \S 2.8,2.7$ to the transformation $T$ considered as defined on the analytic set $E_{1}, T\left(Y_{1}\right)$ and $Y_{1}$ are analytic sets and $X_{1}$ is the difference of two analytic sets. Since

$$
T\left(X_{1}\right)=\underset{p^{*}}{E}\left\{p^{*} \in T\left(E_{1}\right), N\left(p^{*}, T, E_{1}\right)=1\right\},
$$

$T$ is univalent on $X_{1}$. Since

$$
T\left(Y_{1}\right)=\underset{p^{*}}{E}\left\{p^{*} \in T\left(E_{1}\right), N\left(p^{*}, T, E_{1}\right)>1\right\},
$$

$T\left(X_{1}\right)=T\left(E_{1}\right)-T\left(Y_{1}\right)$. Hence $T\left(X_{1}\right)$ is the difference of two analytic sets.

Set $B_{2}=A \cdot \mathcal{C} X_{1}=B_{1} \cdot \mathcal{C} X_{1}=B_{1} \cdot \mathcal{C} \cdot S_{1}+Y_{1} \cdot B_{2}$ is an analytic set. Set $E_{2}=B_{2} S_{2}$ $+A_{\infty}$,

$$
Y_{2}=\underset{p}{E}\left\{p \in E_{2}, N\left[p^{*}=t(p), T, E_{2}\right]>1\right\},
$$

$X_{2}=E_{2}-Y_{2}$. By the same reasoning as used above, $T$ is univalent on $X_{2}, X_{2}$ is the difference of two analytic sets, and $T\left(X_{2}\right)=T\left(E_{2}\right)-T\left(Y_{2}\right)$ is the difference of two analytic sets.

Continuing in this manner, at the $n$th stage, set $B_{n}=A \cdot \mathcal{C}\left(X_{1}+\ldots\right.$ $\left.+X_{n-1}\right)$. Then $B_{n}=A \cdot \mathcal{C}\left(X_{1}+\cdots+X_{n-2}\right) \cdot \mathcal{C} X_{n-1}=B_{n-1} \mathcal{C} X_{n-1}=B_{n-1} \mathcal{C} S_{n-1}$ 
$+Y_{n-1}$ is an analytic set. Set $E_{n}=B_{n} S_{n}+A_{\infty}$,

$$
Y_{n}=\underset{p}{E}\left\{p \in E_{n}, N\left[p^{*}=t(p), T, E_{n}\right]>1\right\},
$$

$X_{n}=E_{n}-Y_{n}$. By the same reasoning, $T$ is univalent on $X_{n}, X_{n}$ is the difference of two analytic sets, and $T\left(X_{n}\right)=T\left(E_{n}\right)-T\left(Y_{n}\right)$ is the difference of two analytic sets.

The sets $X_{n}$ thus defined satisfy conditions (4) and (5). Since $X_{n} \subset B_{n}$ $\mathcal{C}\left(X_{1} \cdots X_{n-1}\right)$ for each integer $n, X_{i} X_{j}=0, i \neq j$. Thus condition (3) is satisfied. For each integer $n, X_{n} Y_{n}=0$ and $Y_{n} \supset A_{\infty}$. Hence condition (2) is satisfied.

Let $p_{0}$ be any point in $A-A_{\infty}$. Then $N\left[p_{0}^{*}=t\left(p_{0}\right), T, A\right]$ is finite and there is a first sphere $S_{n}$ containing $p_{0}$ and no other point of $T^{-1}\left[t\left(p_{0}\right)\right]$. We assert that $p_{0} \in X_{1}+\cdots+X_{n}$. Assume $p_{0} \in X_{1}+\cdots+X_{n-1}$. Then $p_{0} \in B_{n}$ and, since $p_{0} \in S_{n}$, we have $p_{0} \in E_{n}$. Since $N\left[p_{0}{ }^{*}=t\left(p_{0}\right), T, E_{n}\right]=1, p \in X_{n}$. Thus $p_{0} \in X_{1}+\cdots+X_{n}$ and $A=A_{\infty}+X_{1}+\cdots+X_{n}+\cdots$. Hence condition (1) is satisfied.

2.10. THEOREM. Under the same assumptions as $\$ 2.9$, if $B$ is an analytic set, then for each set $X_{n}$ defined in $\$ 2.9$, the sets $B X_{n}$ and $T\left(B X_{n}\right)$ are the difference of two analytic sets.

Proof. For each integer $n$ (see §2.9), $B X_{n}=B E_{n}-B Y_{n}$ is the difference of two analytic sets. $T\left(B E_{n}\right)$ and $T\left(B Y_{n}\right)$ are analytic sets and $T\left(B E_{n}\right)$ $=T\left(B X_{n}\right)+T\left(B Y_{n}\right)$. Since $T\left(B X_{n}\right) \cdot T\left(B Y_{n}\right)=0, \quad T\left(B X_{n}\right)=T\left(B E_{n}\right)$ $-T\left(B Y_{n}\right)$. Hence $T\left(B X_{n}\right)$ is the difference of two analytic sets.

\section{Chapter III. Transformation formulas}

3.1. Throughout this chapter $M$ will be a complete and separable metric space and $M^{*}$ will be a metric space. $\nu(E)$ and $\nu^{*}\left(E^{*}\right)$ will be finite Carathéodory outer measures which are regular with respect to open sets (see $\S \S 1.17,1.19)$ defined on $M$ and $M^{*}$ respectively. Sets measurable (see $\$ 1.17$ ) with respect to $\nu\left(\nu^{*}\right)$ will be called $\nu$-measurable $\left(\nu^{*}\right.$-measurable) sets.

3.2. For a given single-valued continuous transformation $T$ from an analytic set $A \subset M$ onto a (necessarily) analytic set $A^{*} \subset M^{*}$ (see $\$ 2.1$ ) we define a class $\mathcal{X}(T)$ of subsets of $M$ as follows. A set $E \in \mathcal{X}(T)$ if and only if, for each set $X_{n}, n=1,2, \ldots$, defined in $\$ 2.9$, the set $T\left(E X_{n}\right)$ is a $\nu^{*}$-measurable set. While we shall work with a fixed decomposition of $A$ for a given transformation $T$, we shall see in $\$ 3.4$ that $\mathcal{X}(T)$ is independent of the decomposition of $A$ into sets having the properties stated in \$2.9.

3.3. Lemma. The class $\mathcal{X}(T)$ defined in $\$ 3.2$ is a completely additive class of sets (see \$1.12) containing all analytic sets.

Proof. The empty set obviously belongs to $\mathscr{X}(T)$.

Since $E X_{n}+X_{n} \mathcal{C} E=X_{n}$ and $T\left(E X_{n}\right) \cdot T\left(X_{n} \mathcal{C} E\right)=0$ for $n=1,2, \cdots$, if 
$E \in \mathcal{X}(T)$, then $T\left(X_{n} \mathcal{C} E\right)=T\left(X_{n}\right)-T\left(E X_{n}\right)$ is a $\nu^{*}$-measurable set. Hence $\mathcal{C} E \in \mathcal{H}(T)$.

If $E_{m} \in \mathcal{X}(T)$ for $m=1,2, \cdots$, then $T\left(E_{m} X_{n}\right)$ is a $\nu^{*}$-measurable set for every pair of integers $m, n$. If $E=E_{1}+\cdots+E_{m}+\cdots$, then for each integer $n, \quad T\left(E X_{n}\right)=T\left[\left(E_{1}+\cdots+E_{m}+\cdots\right) \cdot X_{n}\right]=T\left(E_{1} X_{n}\right)+\cdots$ $+T\left(E_{m} X_{n}\right)+\cdots$ and $T\left(E X_{n}\right)$ is a $\nu^{*}$-measurable set. Hence $E \in \mathcal{X}(T)$.

By $\$ 2.10$, for each analytic set $B$, the sets $T\left(B X_{n}\right), n=1,2, \cdots$, are the difference of two analytic sets and hence are $\nu^{*}$-measurable. Thus $B \in \mathcal{X}(T)$.

Therefore, $\mathcal{X}(T)$ is a completely additive class of sets containing all analytic sets.

3.4. We shall now show that the class $\mathcal{H}(T)$ defined in $\S 3.2$ is independent of the decomposition of $A$ into sets having the properties listed in \$2.9. Let $A=A_{\infty}+X_{1}^{\prime}+\cdots+X_{n}^{\prime}+\cdots$ and $A=A_{\infty}+X_{1}^{\prime \prime}+\cdots+X_{n}^{\prime \prime}+\cdots$ be two decompositions of $A$ where the sets $X_{n}^{\prime}$ and $X_{n}^{\prime \prime}$ satisfy the condition of $\S 2.9$. Let $\mathcal{H}^{\prime}(T)$ and $\mathcal{X}^{\prime \prime}(T)$ be the classes of subsets determined by these decompositions by the definition of $\S 3.2$. Since, by $\S 3.3, \mathcal{X}^{\prime}(T)$ is a completely additive class of sets containing all the analytic sets, each set $X_{n}^{\prime \prime}$ $\in \mathcal{X}^{\prime}(T)$. For a set $E \in \mathcal{X}^{\prime}(T)$ we have (since $X_{n}^{\prime \prime} \subset X_{1}^{\prime}+\cdots+X_{m}^{\prime}$ $+\cdots) E X_{n}^{\prime \prime}=\left(E X_{1}^{\prime}+\cdots+E X_{m}^{\prime}+\cdots\right) X_{n}^{\prime \prime}=E X_{n}^{\prime \prime} X_{1}^{\prime}+\cdots$ $+E X_{n}^{\prime \prime} X_{m}^{\prime}+\cdots$ and $T\left(E X_{n}^{\prime \prime}\right)=T\left(E X_{n}^{\prime \prime} X_{1}^{\prime}\right)+\cdots+T\left(E X_{n}^{\prime \prime} X_{m}^{\prime}\right)$ $+\cdots$. Since $E$ and $X_{n}^{\prime \prime}$ are in $\mathcal{H}^{\prime}(T)$, the set $E X_{n}^{\prime \prime} \in \mathcal{H}^{\prime}(T)$. Thus $T\left(E X_{n}^{\prime \prime}\right)$ is the sum of a denumerable number of $\nu^{*}$-measurable sets and hence is itself a $\nu^{*}$-measurable set. Thus $E \in \mathcal{H}^{\prime \prime}(T)$. Therefore, $\mathcal{H}^{\prime}(T) \subset \mathcal{H}^{\prime \prime}(T)$. Similarly $\mathcal{H}^{\prime \prime}(T) \subset \mathcal{H}^{\prime}(T)$ and hence $\mathcal{H}^{\prime}(T) \equiv \mathcal{H}^{\prime \prime}(T)$.

3.5. Lemma. If $E^{*}$ is a $\nu^{*}$-measurable set in $M^{*}$ and $E=T^{-1}\left(E^{*}\right)$, then $E \in \mathcal{H}(T)$.

Proof. Since $E$ is the inverse of $E^{*}, T\left(E X_{n}\right)=T\left(X_{n}\right) \cdot T(E)$ for $n=1,2, \cdots$. Thus for each integer $n, T\left(E X_{n}\right)$ is the product of two $\nu^{*}$-measurable sets and hence is itself a $\nu^{*}$-measurable set. Therefore, $E \in \mathcal{X}(T)$.

3.6. For each set $E \in \mathcal{X}(T)$ (see $\$ 3.2$ ) we define the set function

$$
\mu(E, T)=\sum_{n=1}^{\infty} \nu^{*}\left[T\left(E X_{n}\right)\right]
$$

Theorem. $\mu(E, T)$ is a measure on $\mathscr{H}(T)$ (see $\$ 1.16)$.

Proof. $\mathcal{X}(T)$ is a completely additive class of sets by $\S 3.3$ and $\mu(E, T)$ is defined and non-negative for sets $E \in \mathcal{X}(T)$. Let $E=E_{1}+\cdots+E_{n}+\cdots$, $E_{n} \in \mathcal{H}(T), n=1,2, \cdots, E_{i} E_{j}=0, i \neq j$. Since $T$ is univalent on $X_{n}$, for each integer $n$

Thus

$$
\nu^{*}\left[T\left(E X_{n}\right)\right]=\sum_{m=1}^{\infty} \nu^{*}\left[T\left(E_{m} X_{n}\right)\right]
$$




$$
\mu(E, T)=\sum_{n=1}^{\infty} \nu^{*}\left[T\left(E X_{n}\right)\right]=\sum_{n=1}^{\infty} \sum_{m=1}^{\infty} \nu^{*}\left[T\left(E_{m} X_{n}\right)\right]=\sum_{m=1}^{\infty} \mu\left(E_{m}, T\right) .
$$

3.7. Let $\Phi$ be a measure on a completely additive class of subsets of an abstract space 1 . Sets in this completely additive class of sets will be called $\Phi$-measurable sets. A real-valued function $f(p)$ defined on a $\Phi$-measurable set $E$ is called a $\Phi$-measurable function on $E$ if, for every real number $a$, the set

$$
E_{a}=\underset{p}{E}\{f(p)>a, p \in E\}
$$

is a $\Phi$-measurable set.

Let $f(p)$ be a non-negative, $\Phi$-measurable function defined on a $\Phi$-measurable set $E$. Let $m\left(f, E^{\prime}\right)$ denote the greatest lower bound of $f(p)$ for points $p \in E^{\prime}$. The definite integral of $f(p)$ over $E$ is defined to be

$$
\int_{E} f(p) d \Phi=\text { l.u.b. }\left[m\left(f, E_{1}\right) \Phi\left(E_{1}\right)+\cdots+m\left(f, E_{k}\right) \Phi\left(E_{k}\right)\right],
$$

where the least upper bound is taken with respect to all finite sequences $E_{1}, \cdots, E_{k}$ of $\Phi$-measurable sets such that $E=E_{1}+\cdots+E_{k}, E_{i} E_{j}=0, i \neq j$.

For an arbitrary $\Phi$-measurable function $f(p)$ defined on a $\Phi$-measurable set $E$, let $f_{1}(p)=f(p)$ if $f(p) \geqq 0$ and $f_{1}(p)=0$ if $f(p)<0$, and let $f_{2}(p)=f(p)$ if $f(p)<0$ and $f_{2}(p)=0$ if $f(p) \geqq 0$. If the definite integral of either $f_{1}(p)$ or $-f_{2}(p)$ is finite on $E$, the definite integral of $f(p)$ over $E$ is defined to be the definite integral of $f_{1}(p)$ over $E$ minus the definite integral of $-f_{2}(p)$ over $E$.

If the definite integral of a $\Phi$-measurable function $f(p)$ is finite over a $\Phi$-measurable set $E, f(p)$ is called a $\Phi$-summable function on $E$.

For the properties of measurable functions and definite integrals used in the sequel, the reader is referred to Saks [5, chap. I].

3.8. Lemma. For each set $E \in \mathcal{H}(T)$, the function $N\left(p^{*}, T, E-A_{\infty}\right)$ is a finite-valued and $\nu^{*}$-measurable function (see $\$ 2.1,2.8,3.7$ ).

Proof. Since $N\left(p^{*}, T, E-A_{\infty}\right) \leqq N\left(p^{*}, T, A-A_{\infty}\right)$ for every point $p^{*} \in M^{*}$, $N\left(p^{*}, T, E-A_{\infty}\right)$ is a finite-valued function.

Since $T$ is univalent on each set $X_{n}, N\left(p^{*}, T, E X_{n}\right)=1$ for $p^{*} \in T\left(E X_{n}\right)$ and $N\left(p^{*}, T, E X_{n}\right)=0$ for $p^{*} \notin T\left(E X_{n}\right)$. Since $E \in \mathcal{X}(T), T\left(E X_{n}\right)$ is a $\nu^{*}$ measurable set and hence $N\left(p^{*}, T, E X_{n}\right)$ is a $\nu^{*}$-measurable function for $n=1,2, \cdots$ Hence $N\left(p^{*}, T, E-A_{\infty}\right)=N\left(p^{*}, T, E X_{1}\right)+\cdots+N\left(p^{*}, T\right.$, $\left.E X_{n}\right)+\cdots$ is a $\nu^{*}$-measurable function (see Saks [5, chap. I, Theorem 12.3]).

3.9. Lemma. For each set $E \in \mathcal{X}(T)$

$$
\mu(E, T)=\int_{M^{*}} N\left(p^{*}, T, E-A_{\infty}\right) d \nu^{*} .
$$

Proof. The proof follows from Theorem 12.3, Chapter I, Saks [5], since 
$\left(N p^{*}, T, E-A_{\infty}\right)=N\left(p^{*}, T, E X_{1}\right)+\cdots+N\left(p^{*}, T, E X_{n}\right)+\cdots$ and $\mu\left(E X_{n}, T\right)=\nu^{*}\left[T\left(E X_{n}\right)\right]=\int_{M} N\left(p^{*}, T, E X_{n}\right) d \nu^{*}$ for $n=1,2, \cdots$.

3.10. Lemma. If $H\left(p^{*}\right)$ is a finite-valued function defined on $A^{*}-A_{\infty}^{*}$, then $H\left(p^{*}\right)$ is a $\nu^{*}$-measurable function if and only if $H[t(p)]$ is a $\mu$-measurable function on $A-A_{\infty}$.

Proof. For any real number $a$,

$$
E_{a}=\underset{p}{E}\{H[t(p)]>a\}=T^{-1}\left(E_{a}^{*}\right), \text { where } E_{a}^{*}=\underset{p^{*}}{E^{*}}\left\{H\left(p^{*}\right)>a\right\} .
$$

By $\S \S 3.2$ and $3.5, E_{a}^{*}$ is a $\nu^{*}$-measurable set if and only if $E_{a}$ is a $\mu$-measurable set.

3.11. Let $f(p)$ be a finite-valued function defined on $A$. For each set $E \subset M$ and set $X_{n}$ (see \$2.9) we define

$$
\begin{aligned}
& \sigma_{n}\left(p^{*}, T, E, f\right)=f(p) \quad \text { if } \quad p=T^{-1}\left(p^{*}\right) \cdot E X_{n} \quad \text { and } \\
& \sigma_{n}\left(p^{*}, T, E, f\right)=0 \quad \text { if } \quad p^{*} \notin T\left(E X_{n}\right), \\
& \sigma\left(p^{*}, T, E, f\right)=\sum_{n=1}^{\infty} \sigma_{n}\left(p^{*}, T, E, f\right) .
\end{aligned}
$$

3.12. We have the following identities and relationships for the functions defined in $\$ 3.11$. Let $H\left(p^{*}\right)$ be any finite-valued function defined on $M^{*}$.

(i) Since $T$ is univalent on $X_{n}, n=1,2, \cdots, H\left(p^{*}\right)=\sigma_{n}\left(p^{*}, T, E, H[t(p)]\right)$, $p^{*} \in T\left(E X_{n}\right)$.

(ii) From (i) we have, since $N\left(p^{*}, T, E\right)=\sigma\left(p^{*}, T, E, f\right)=0$ for $p^{*} \notin\left(A^{*}\right.$ $\left.-A_{\infty}^{*}\right) \cdot T(E)+A_{\infty}^{*}, H\left(p^{*}\right) N\left(p^{*}, T, E\right)=\sigma\left(p^{*}, T, E, H[t(p)]\right), p^{*} \in M^{*}-A_{\infty}^{*}$.

(iii) Let $f(p)$ be a finite-valued function defined on $A$. Then for each integer $n, H\left(p^{*}\right) \sigma_{n}\left(p^{*}, T, E, f\right)=\sigma_{n}\left(p^{*}, T, E, H[t(p)] f(p)\right), p^{*} \in A^{*}$.

(iv) From (iii) we have $H\left(p^{*}\right) \sigma\left(p^{*}, T, E, f\right)=\sigma\left(p^{*}, T, E, H[t(p)] f(p)\right)$, $p^{*} \in A^{*}$.

(v) Since $\sigma_{n}(t(p), T, E, f)=f(p), p \in E X_{n}$, and $\sigma_{n}(t(p), T, E, f)=0, p \notin E X_{n}$, it follows from $\$ 3.10$ that $\sigma_{n}\left(p^{*}, T, E, f\right)$ is a $\nu^{*}$-measurable function if $E X_{n}$ is a $\mu$-measurable set and $f$ is a $\mu$-measurable function on $E X_{n}$.

(vi) From (v) it follows that $\sigma\left(p^{*}, . T, E, f\right)$ is a $\nu^{*}$-measurable function if $E$ is a $\mu$-measurable set and $f$ is a $\mu$-measurable function on $E$.

3.13. Lemma. If $f(p)$ is any non-negative, finite-valued, $\mu$-measurable function defined on $A$, then, for any $\mu$-measurable set $E$ and any integer $n$,

$$
\int_{E X_{n}} f(p) d \mu=\int_{M^{*}} \sigma_{n}\left(p^{*}, T, E, f\right) d \nu^{*} .
$$

Proof. (a) From (v) of $\$ 3.12, \sigma_{n}\left(p^{*}, T, E, f\right)$ is a $\nu^{*}$-measurable function. Since $\sigma_{n}\left(p^{*}, T, E, f\right)=0$ for $p^{*} \in T\left(E X_{n}\right)$, the integral on the right can be considered as taken over the set $T\left(E X_{n}\right)$. 
(b) If $E^{\prime}$ is any subset of $E X_{n}$, then (see $\left.\S 3.7\right) m\left(f, E^{\prime}\right)=m\left[\sigma_{n}\left(p^{*}, T, E, f\right)\right.$, $\left.T\left(E^{\prime}\right)\right]$.

(c) If $E^{\prime}$ is any $\mu$-measurable subset of $E X_{n}$, then (see $\left.\S \S 3.2,3.6\right), T\left(E^{\prime}\right)$ is a $\nu^{*}$-measurable set and $\mu\left(E^{\prime}\right)=\nu^{*}\left[T\left(E^{\prime}\right)\right]$.

(d) If $E X_{n}=E_{1}+\cdots+E_{k}, E_{i} E_{j}=0, i \neq j$, where $E_{1}, \cdots, E_{k}$ are $\mu$ measurable sets, then $T\left(E X_{n}\right)=T\left(E_{1}\right)+\cdots+T\left(E_{k}\right), T\left(E_{i}\right) \cdot T\left(E_{j}\right)=0, i \neq j$, and the sets $T\left(E_{1}\right), \cdots, T\left(E_{k}\right)$ are $\nu^{*}$-measurable sets. This follows from the univalence of $T$ on $E X_{n}$ and the definition of $\mu$-measurable sets.

(e) If $T\left(E X_{n}\right)=E_{1}^{*}+\cdots+E_{k}^{*}, E_{i}{ }^{*} E_{j}^{*}=0, i \neq j$, where $E_{1}^{*}, \cdots, E_{k}^{*}$ are $\nu^{*}$-measurable sets, then for $E_{i}=E X_{n} \cdot T^{-1}\left(E_{i}^{*}\right), i=1, \cdots, k$, we have $E X_{n}=E_{1}+\cdots+E_{k}, E_{i} E_{j}=0, i \neq j$ and the sets $E_{i}$ are $\mu$-measurable sets. This follows from the definition of the inverse of a set and the definition of $\mu$-measurable sets.

The lemma then follows from (a), (b), (c), (d), (e) and the definition of the definite integral (see \$3.7).

3.14. Theorem. If $f(p)$ is any non-negative, finite-valued, $\mu$-measurable function defined on $M$, then for any $\mu$-measurable set $E$,

$$
\int_{E} f(p) d \mu=\int_{M^{*}} \sigma\left(p^{*}, T, E, f\right) d \nu^{*} .
$$

Proof. Since $\mu\left[\mathcal{C}\left(A-A_{\infty}\right), T\right]=0$, we have (see Saks [5, Theorem 12.8, chap. I])

$$
\int_{E} f(p) d \mu=\sum_{n=1}^{\infty} \int_{E X_{n}} f(p) d \mu .
$$

By (vi) of $\$ 3.12$, and by $\S 3.10, \sigma\left(p^{*}, T, E, f\right)$ is a $\nu^{*}$-measurable function. Since each $\sigma_{n}\left(p^{*}, T, E, f\right) \geqq 0$ and $\sigma\left(p^{*}, T, E, f\right)=\sigma_{1}\left(p^{*}, T, E, f\right)+\cdots$ $+\sigma_{n}\left(p^{*}, T, E, f\right)+\cdots$ we have (see Saks [5, Theorem 12.3, chap. I])

$$
\int_{M^{*}} \sigma\left(p^{*}, T, E, f\right) d \nu^{*}=\sum_{n=1}^{\infty} \int_{M^{*}} \sigma_{n}\left(p^{*}, T, E, f\right) d \nu^{*} .
$$

The theorem follows from (1), (2) and $\S 3.13$.

3.15. Theorem. If $f(p)$ is a finite-valued, $\mu$-measurable function defined on $M$, then, for any $\mu$-measurable set $E$,

$$
\int_{E} f(p) d \mu=\int_{M^{*}} \sigma\left(p^{*}, T, E, f\right) d \nu^{*}
$$

whenever the integral on the left exists.

Proof. Assume that the integral on the left exists. The sets

$$
E_{1}=\underset{p}{E}[f(p) \geqq 0, p \in E], \quad E_{2}=\underset{p}{E}[f(p)<0, p \in E]
$$


are $\mu$-measurable sets. By $\$ 3.14$,

$$
\int_{E_{1}} f(p) d \mu=\int_{M^{*}} \sigma\left(p^{*}, T, E_{1}, f\right) d v^{*}, \quad \int_{E_{2}} f(p) d \mu=\int_{M^{*}} \sigma\left(p^{*}, T, E_{2}, f\right) d \nu^{*}
$$

At least one of these integrals is finite by assumption. Hence the statement of the theorem follows.

That the existence of the integral on the right side does not in general imply the existence of the integral on the left side can be shown by the following example. Let $A$ consist of two disjoint, closed, congruent squares $s_{1}, s_{2}$ in the $u v$-plane and let $s^{*}$ be a third congruent square in the $x y$-plane. The transformation $T$ consists of mating each of the squares $s_{1}, s_{2}$ congruently onto $s^{*}$. Then $T$ is a single-valued, continuous transformation from $A=s_{1}+s_{2}$ onto $A^{*}=s^{*}$. The sets $X_{1}, X_{2}$ can be taken as $s_{1}, s_{2}$ respectively and the sets $X_{n}, n=3,4, \cdots$, as the empty sets. Let $f(u, v)$ be a function which is nonnegative, Lebesgue measurable and has an infinite definite integral on $s_{1}$ and takes on corresponding negative values at corresponding points of $s_{2}$. If $\nu^{*}$ is taken as the Lebesgue exterior measure in the $x y$-plane then $\mu(E, T)$ is the Lebesgue measure of Lebesgue measurable subsets of $A$ in the $u v$-plane. Hence $f(u, v)$ is a $\mu$-measurable function but does not have a definite integral on $A$. However, $\sigma\left(p^{*}, T, A, f\right) \equiv 0$.

3.16. Theorem. If $H\left(p^{*}\right)$ is a finite-valued, $\nu^{*}$-measurable function defined on $M^{*}$ and $g(p)$ is any finite-valued, $\mu$-measurable function defined on $M$, then, for any $\mu$-measurable set $E \subset A$,

$$
\int_{E} H[t(p)] g(p) d \mu=\int_{M^{*}} H\left(p^{*}\right) \sigma\left(p^{*}, T, E, g\right) d \nu^{*}
$$

whenever the integral on the left exists. If $g(p) \geqq 0$, then the relation holds whenever the integral on the right exists.

Proof. By $\S 3.10, H[t(p)]$ is a $\mu$-measurable function on $A-A_{\infty}$. By the definition of $\mathcal{X}(T)$, every subset of $A_{\infty}$ is in $M(T)$. Hence $H[t(p)]$ is a $\mu$ measurable function on $A$. The first part of the theorem follows from $\$ 3.15$ for $f(p)=H[t(p)] g(p)$ and the identity (iv) of $\$ 3.12$.

If the integral on the right side exists then at least one of the the integrals

where

$$
\int_{M^{*}} H\left(p^{*}\right) \sigma\left(p^{*}, T, E_{1}, g\right) d \nu^{*}, \quad \int_{M^{*}} H\left(p^{*}\right) \sigma\left(p^{*}, T, E_{2}, g\right) d \nu^{*},
$$

$$
E_{1}=\underset{p}{E}\{H[t(p)] \geqq 0, p \in E\}, \quad E_{2}=\underset{p}{E}\{H[t(p)]<0, p \in E\}
$$

is finite. By applying $\S 3.14$ to $f(p)=H[t(p)] g(p)$ on $E_{1}$ and $E_{2}$, the integral on the left exists.

3.17. Let $T$ be a single-valued, continuous transformation from an analytic set $A \subset M$ onto a set $A^{*} \subset M^{*}$. The function $N\left(p^{*}, T, A\right)$ (see $\left.\S \S 2.1,2.8\right)$ 
is a $\nu^{*}$-measurable function defined on $M^{*}$. We shall say that $T$ is of bounded variation on $A$, briefly, B.V. on $A$, if $N\left(p^{*}, T, A\right)$ is a $\nu^{*}$-summable function on $M^{*}$ (see $\left.\S 3.7\right)$.

3.18. Theorem. Let T be B.V. on A. If $H\left(p^{*}\right)$ is any finite-valued, $\nu^{*}$ measurable function defined on $M^{*}$, then, for any $\mu$-measurable set $E \subset A$,

$$
\int_{E} H[t(p)] d \mu=\int_{M^{*}} H\left(p^{*}\right) N\left(p^{*}, T, E\right) d \nu^{*},
$$

provided only that one of the two integrals involved exists.

Proof. By (ii) of $\$ 3.12, H\left(p^{*}\right) N\left(p^{*}, T, E\right)=\sigma\left(p^{*}, T, E, H[t(p)]\right)$ except on $A_{\infty}{ }^{*}$. Since $N\left(p^{*}, T, A\right)$ is a $\nu^{*}$-summable function, the set of points $A_{\infty}{ }^{*}$ where $N\left(p^{*}, T, A\right)$ is infinite is of $\nu^{*}$-measure zero (see, Saks [5, Theorem 11.8, chap. I]). The theorem follows then from $\$ 3.16$.

3.19. Lemma. If $T$ is B.V. on $A$, then, by $\S 3.9, \mu(E, T)$ is a finite measure (completely additive set function) on $\mathscr{H C}(T)$.

3.20. The class $\mathcal{H}(T)$ of $\mu$-measurable sets and the class of $\nu$-measurable sets (see $\$ 3.1$ ) are not in general the same class of sets. However, by $\S 3.2$ and $\S \S 1.17,1.18$, both of these classes are families of sets $K(\mathcal{S}, \Sigma, \mathcal{C}, \Pi, D$ ) (see $\S 1.10)$, where $S$ is the class of analytic subsets of $M$. Hence the product of these two classes, which we shall denote by $\mathscr{H}_{0}(T)$, is a family of sets $K(\mathcal{S}, \Sigma$, $\mathcal{C}, \Pi, D)$.

3.21. Assume $T$ is B.V. on $A$. By the Lebesgue decomposition of a completely additive set function (see Saks [5, Theorem 14.6, chap. I]) the set function $\mu(E, T)$ can be expressed uniquely as the sum of two non-negative completely additive set functions

$$
\mu(E, T)=\mu_{a}(E, T)+\mu_{s}(E, T), \quad E \in \mathcal{X}_{0}(T),
$$

where $\mu_{a}(E, T)$ is an absolutely continuous set function with respect to $\nu$ and $\mu_{s}(E, T)$ is a singular set function with respect to $\nu$ on $\mathcal{X}_{0}(T)$. That is to say, $\mu_{a}(E, T)=0$ for every set $E \in \mathcal{X}_{0}(T)$ of $\nu$-measure zero and there exists a set $e_{s}$ (called the singular set) in $\mathcal{X}_{0}(T)$ of $\nu$-measure zero such that $\mu_{s}\left(C e_{s}, T\right)=0$.

By the Theorem of Radon-Nikodym (see Saks [5, Theorem 14.11, chap. I]) the absolutely continuous set function $\mu_{a}(E, T)$ can be expressed as the $\nu$-integral of a non-negative, finite-valued function $D(p, T)$ which is both $\mu$-measurable and $\nu$-measurable. Thus we can write

$$
\mu(E, T)=\int_{E} D(p, T) d \nu+\mu_{s}(E, T), \quad E \in \mathcal{X}_{0}(T) .
$$

3.22. Lemma. Assume $T$ is B.V. on A. Let $e_{s}$ be the singular set (see \$3.21). If $E \subset C e_{s}$ is a $\nu$-measurable set then $E$ is a $\mu$-measurable set. 
Proof. Since $\nu$ is regular with respect to open sets (see \$1.19), for each integer $n$ we have Borel sets $B_{n}^{\prime}, B_{n}^{\prime \prime}$ such that $B_{n}^{\prime} \subset E X_{n} \subset B_{n}{ }^{\prime \prime} X_{n} \mathcal{C} e_{8} \subset B_{n}{ }^{\prime \prime}$ and $\nu\left(B_{n}^{\prime}\right)=\nu\left(E X_{n}\right)=\nu\left(B_{n}^{\prime \prime}\right)=\nu\left(B_{n}^{\prime \prime} X_{n} \mathcal{C} e_{s}\right)$. The sets $B_{n}^{\prime}, B_{n}^{\prime \prime} X_{n} C e_{s}$ are $\mu$ measurable sets. Since $\nu\left(B_{n}^{\prime \prime} X_{n} \mathcal{C} e_{s}-B_{n}^{\prime}\right)=0$ and $\left(B_{n}^{\prime \prime} X_{n} \mathcal{C} e_{s}-B_{n}^{\prime}\right) \subset \mathcal{C} e_{s}$, we have that $\mu\left(B_{n}^{\prime \prime} X_{n} C e_{s}-B_{n}^{\prime}, T\right)=0$ and $\mu\left(B_{n}^{\prime}, T\right)=\mu\left(B_{n}^{\prime \prime} X_{n} C e_{s}, T\right)$. Since $T\left(B_{n}^{\prime}\right) \subset T\left(E X_{n}\right) \subset T\left(B_{n}^{\prime \prime} X_{n} \mathcal{C} e_{s}\right)$ and $\nu^{*}\left[T\left(B_{n}^{\prime}\right)\right]=\mu\left(B_{n}^{\prime}, T\right)=\mu\left(B_{n}^{\prime \prime} X_{n} \mathcal{C} e_{s}, T\right)$ $=\nu^{*}\left[T\left(B_{n}^{\prime \prime} X_{n}\left(e_{s}\right)\right]\right.$, the set $T\left(E X_{n}\right)$ is $\nu^{*}$-measurable. Hence $E X_{n}$ is $\mu$-measurable for each integer $n$ and $E$ is a $\mu$-measurable set.

3.23. Lemma. If $E$ is a $\mu$-measurable set, then there exist sets $E_{1}, E_{2} \in \mathcal{H}_{0}(T)$ such that $E_{1} \subset E \subset E_{2}$ and $\mu\left(E_{1}, T\right)=\mu(E, T)=\mu\left(E_{2}, T\right)$.

Proof. For each integer $n$, the set $T\left(E X_{n}\right)$ is a $\nu^{*}$-measurable set. Since $\nu^{*}$ is regular with respect to open sets, we have Borel sets $B_{1, n}^{*}, B_{2, n}^{*}$ such that $B_{1, n}^{*} \subset T\left(E X_{n}\right) \subset B_{2, n}^{*}$ and $\nu^{*}\left(B_{1, n}^{*}\right)=\nu^{*}\left[T\left(E X_{n}\right)\right]=\nu^{*}\left(B_{2, n}^{*}\right)$. By $\$ 2.7, T^{-1}\left(B_{1, n}^{*}\right)$ and $T^{-1}\left(B_{2, n}^{*}\right)$ are analytic sets. Hence, the sets $E_{1, n}=X_{n} T^{-1}\left(B_{1, n}^{*}\right)$ and $E_{2, n}=X_{n} T^{-1}\left(B_{2, n}^{*}\right)$ are sets in $\mathcal{X}_{0}(T)$ and $E_{1, n} \subset E X_{n} \subset E_{2, n}$. Furthermore, $B_{1, n}^{*}=T\left(E_{1, n}\right) \subset T\left(E X_{n}\right) \subset T\left(E_{2, n}\right) \subset B_{2, n}^{*}$. Hence $\mu\left(E_{1, n}, T\right)=\mu\left(E X_{n}, T\right)$ $=\mu\left(E_{2, n}, T\right)$. Since $\mathcal{C}\left(A-A_{\infty}\right) \in \mathcal{X}_{0}(T)$ and $\mu\left[\mathcal{C}\left(A-A_{\infty}\right), T\right]=0$ and since $\mu$ is a measure on $\mathcal{X}_{0}(T)$, the sets $E_{1}=E_{1,1}+\cdots+E_{1, n}+\cdots$ and $E_{2}=\mathcal{C}(A$ $\left.-A_{\infty}\right)+E_{2,1}+\cdots+E_{2, n}+\cdots$ satisfy the conditions of the lemma.

3.24. Lemma. Assume $T$ is B.V. on $A$. If $f(p)$ is a finite-valued, $\mu$-measurable function defined on $M$, then $f(p) D(p, T)$ is a $\nu$-measurable function.

Proof. We may assume that $f(p)$ is non-negative on $M$. Since $f(p)$ and $D(p, T)$ are $\mu$-measurable functions, the product $f(p) D(p, T)$ is a $\mu$-measurable function. For any real number $a>0$, the set

$$
E_{a}=\underset{p}{E}[f(p) D(p, T) \geqq a>0]
$$

is a $\mu$-measurable set. By $\$ 3.23$, we have sets $E_{1}, E_{2} \in \mathcal{X}_{0}(T)$ such that $E_{1} \subset E_{a} \subset E_{2}$ and $\mu\left(E_{1}, T\right)=\mu\left(E_{a}, T\right)=\mu\left(E_{2}, T\right)$. Then $\mu\left(E_{2}-E_{1}, T\right)=\mu\left(E_{2}, T\right)$ $-\mu\left(E_{1}, T\right)=0$. Thus

$$
0=\mu\left(E_{2}-E_{1}, T\right)=\int_{E_{2}-E_{1}} D(p, T) d \nu+\mu_{8}\left(E_{2}-E_{1}, T\right) .
$$

Thus $D(p, T)=0$ on $E_{2}-E_{1}$ except on a set of $\nu$-measure zero. Hence $f(p) D(p, T)=0$ on $E_{a}-E_{1}$ except on a set of $\nu$-measure zero. Since $f(p) D(p, T)$ $\geqq a>0$ at every point of $E_{a}-E_{1}$ we conclude that $E_{a}-E_{1}$ is of $\nu$-measure zero. Hence $E_{a}$ is a $\nu$-measurable set and $f(p) D(p, T)$ is a $\nu$-measurable function.

3.25. Lemma. Assume $T$ is B.V. on $A$. If $f(p)$ is a finite-valued, $\mu$-measurable function defined on $M$, then for any set $E \in \mathcal{H}_{0}(T)$ on which the definite integral of $f(p)$ exists,

$$
\int_{E} f(p) d \mu=\int_{E} f(p) D(p, T) d \nu+\int_{E} f(p) d \mu_{s} .
$$


Proof. Since $\nu\left(e_{s}\right)=0$, where $e_{s}$ is the singular set, it is sufficient to prove the theorem for the case $E \subset \mathcal{C} e_{s}$.

(i) We hrst assume that $f(p) \geqq 0$. Let $\epsilon>0$ be given. Set, for $n=1,2, \cdots$,

$$
E_{n}=\underset{p}{E}[(n-1) \epsilon<f(p) \leqq n \epsilon ; p \in E] .
$$

By $\$ 3.23$, we have sets $B_{n} \in \mathcal{X}_{0}(T)$ such that $B_{n} \subset E_{n}$ and $\mu\left(B_{n}, T\right)=\mu\left(E_{n}, T\right)$. Since $\mu\left(E_{n}-B_{n}, T\right)=0, D(p, T)=0$ on $\left(E_{1}-B_{1}\right)+\cdots+\left(E_{n}-B_{n}\right)+\cdots$ except for a sec of $\nu$-measure zero. We have

$$
\begin{aligned}
\int_{E} f(p) d \mu-\epsilon \mu(E, T) & \leqq \sum_{n=1}^{\infty}(n-1) \epsilon \int_{E_{n}} d \mu=\sum_{n=1}^{\infty}(n-1) \epsilon \int_{B_{n}} d \mu \\
& =\sum_{n=1}^{\infty}(n-1) \epsilon \int_{B_{n}} D(p, T) d \nu \leqq \sum_{n=1}^{\infty} \int_{B_{n}} f(p) D(p, T) d \nu \\
& =\int_{E} f(p) D(p, T) d \nu \leqq \sum_{n=1}^{\infty} n \epsilon \int_{B_{n}} D(p, T) d \nu \\
& =\sum_{n=1}^{\infty} n \epsilon \int_{B_{n}} d \mu=\sum_{n=1}^{\infty} n \epsilon \int_{E_{n}} d \mu \leqq \int_{E} f(p) d \mu+\epsilon \mu(E, T) .
\end{aligned}
$$

Since $\epsilon>0$ is arbitrary,

$$
\int_{E} f(p) d \mu=\int_{E} f(p) D(p, T) d \nu
$$

(ii) Let $f(p)$ be an arbitrary $\mu$-integrable function. Let

$$
E_{1}=\underset{p}{E}[f(p) \geqq 0 ; p \in E], \quad E_{2}=\underset{p}{E}[f(p)<0 ; p \in E] .
$$

By $\S 3.23$, we have sets $B_{1}, B_{2} \in \mathcal{X}_{0}(T)$ such that $B_{1} \subset E_{1}, B_{2} \subset E_{2}, \mu\left(B_{1}, T\right)$ $=\mu\left(E_{1}, T\right), \mu\left(B_{2}, T\right)=\mu\left(E_{2}, T\right)$. Furthermore, $D(p, T)=0$ on $\left(E_{1}-B_{1}\right)$ $+\left(E_{2}-B_{2}\right)$ except on a set of $\nu$-measure zero. Hence by (i)

$$
\begin{aligned}
\int_{E} f(p) d \mu & =\int_{B_{1}} f(p) d \mu+\int_{B_{2}} f(p) d \mu \\
& =\int_{B_{1}} f(p) D(p, T) d \nu+\int_{B_{2}} f(p) D(p, T) d \nu \\
& =\int_{E} f(p) D(p, T) d \nu .
\end{aligned}
$$

3.26. Assume $T$ is B.V. on $A$. By using $\$ 3.25$, the theorems in $\S \S 3.16$, 3.18 can be stated in the following form.

Theorem. Assume Tis B.V. on A. If $f(p)$ is a finite-valued, $\mu$-measurable function defined on $M$ and $H\left(p^{*}\right)$ is a finite-valued, $\nu^{*}$-measurable function de- 
fined on $M^{*}$, then, for any set $E \in \mathcal{X}_{0}(T), E \subset A$,

(a) $\int_{E} H[t(p)] f(p) D(p, T) d \nu+\int_{E} H[t(p)] f(p) d \mu_{s}=\int_{M^{*}} H\left(p^{*}\right) \sigma\left(p^{*}, T, E, f\right) d \nu^{*}$

whenever the two integrals on the left exist but are not both infinite with opposite signs, and, if $f(p) \geqq 0$, whenever the integral on the right exists;

$$
\int_{E} H[t(p)] D(p, T) d \nu+\int_{E} H[t(p)] d \mu_{s}=\int_{M^{*}} H\left(p^{*}\right) N\left(p^{*}, T, E\right) d \nu^{*}
$$

whenever either the two integrals on the left exist but are not both infinite with opposite signs or the integral on the right exists.

3.27. A single-valued, continuous transformation $T: p^{*}=t(p), p \in A \subset M^{\prime}$ $p^{*} \in A^{*} \subset M^{*}$, from an analytic set $A \subset M$ onto a set $A^{*} \subset M^{*}$ which is B.V. on $A$ is called an absolutely continuous transformation on $A$, briefly, A.C. on $A$, if $\nu^{*}[T(E)]=0$ for every set $E \subset A$ of $\nu$-measure zero.

3.28. In general a transformation $T$ such that $\nu^{*}[T(E)]=0$ for every set $E$ of $\nu$-measure zero is not of bounded variation on $A$ as the following example shows. Let $M$ consist of a denumerable number of points $p_{1}, p_{2}, \cdots, p_{n}, \cdots$. Set $\nu\left(p_{n}\right)=1 / 2^{n}$ and $\nu(E)=\sum \nu(p)$, where the summation is taken with respect to all points $p \in E$. Let $M^{*}$ consist of a single point $p^{*}$ and set $\nu^{*}\left(M^{*}\right)=1$. The transformation $T$ consists of mapping $M$ onto $M^{*}$. Since $M$ contains no nonempty set of measure zero, $\nu^{*}[T(E)]=0$ for every set of $\nu$-measure zero. Since $A_{\infty}^{*}=M^{*}, \nu^{*}\left(A_{\infty}^{*}\right)=1$. Thus $T$ is not B.V. on $M$.

3.29. Theorem. Assume $T$ is B.V. on A. A necessary and sufficient condition that $T$ be A.C. on $A$ is that the set function $\mu(E, T)$ be an absolutely continuous completely additive set function on $\mathcal{H}_{0}(T)$.

Proof. Assume that $T$ is A.C. on $A$. Let $E \in \mathcal{A l}_{0}(T)$ be a set of $\nu$-measure zero. Then $\nu^{*}[T(E)]=0$. Hence $N\left(p^{*}, T, E-A_{\infty}\right)=0$ except on a set of $\nu^{*}$-measure zero. By $\$ 3.9, \mu(E, T)=0$. Furthermore, since $\nu(E)=0, \mu(E, T)$ $=\mu_{a}(E, T)+\mu_{s}(E, T)=\mu_{s}(E, T)=0$. Thus $\mu_{s}(E, T)=0$ for every set $E \in \mathcal{X}_{0}(T)$ of $\nu$-measure zero and hence it is equal to zero for every set in $\mathcal{X}_{0}(T)$ (see $\S 3.21)$. Thus $\mu(E, T)=\mu_{a}(E, T)$ is an absolutely continuous completely additive set function on $\mathcal{X}_{0}(T)$.

Assume $\mu(E, T)$ is an absolutely continuous completely additive set function on $\mathcal{H}_{0}(T)$. For any set $E \subset A, E \in \mathscr{X}_{0}(T)$,

$$
\mu_{a}(E, T)=\mu(E, T)=\int_{M^{*}} N\left(p^{*}, T, E-A_{\infty}\right) d \nu^{*} \geqq \nu^{*}\left[T(E)-A_{\infty}^{*}\right] .
$$

If $\nu(E)=0$, then $\mu_{a}(E, T)=0$ and $\nu^{*}\left[T(E)-A_{\infty}\right]=0$. Since $T$ is B.V. on $A$, $\nu^{*}\left(A_{\infty}^{*}\right)=0$. Hence $0=\nu^{*}\left[T(E)-A_{\infty}^{*}\right]+\nu^{*}\left(A_{\infty}^{*}\right) \geqq \nu^{*}[T(E)]$ and $\nu^{*}[T(E)]=0$. 
Let $E^{\prime}$ be any set of $\nu$-measure zero. Since $\nu$ is regular with respect to open sets, we have a Borel set $E \in \mathcal{X}_{0}(T)$ such that $E \supset E^{\prime}, \nu(E)=\nu\left(E^{\prime}\right)=0$. Thus $\nu^{*}\left[T\left(E^{\prime}\right)\right] \leqq \nu^{*}[T(E)]=0$. Thus $T$ is A.C. on $A$.

3.30. Lemma. If a transformation $T$ is B.V. on $A$ and A.C. on $A$, then every $\nu$-measurable set is in $\mathcal{H}_{0}(T)$.

Proof. By $\S 3.29, \mu_{s}(E, T)=0$ for every set $E \in \mathcal{X}_{0}(T)$. Hence the singular set $e_{s}$ (see \$3.21) can be taken as the empty set. The lemma then follows from $\$ 3.22$ and the definition of the class $\mathcal{X}_{0}(T)$ (see $\$ 3.20$ ).

3.31. If a transformation $T$ is B.V. on $A$ and A.C. on $A$, then $\mu_{s}(E, T)=0$ for every set $E \in \mathcal{X}_{0}(T)$. Thus, from $\$ 3.26$ and $\$ 3.30$ we have the fundamental transformation formulas stated in the following theorem.

Theorem. Assume that $T$ is B.V. on $A$ and A.C. on $A$. If $f(p)$ is a finitevalued, $\nu$-measurable function defined on $M$ and $H\left(p^{*}\right)$ is a finite-valued, $\nu^{*}$-measurable function defined on $M^{*}$, then, for any $\nu$-measurable set $E \subset A$

$$
\int_{E} H[t(p)] f(p) D(p, T) d \nu=\int_{M^{*}} H\left(p^{*}\right) \sigma\left(p^{*}, T, E, f\right) d \nu^{*},
$$

whenever the integral on the left exists, and, if $f(p) \geqq 0$, whenever the integral on the right exists;

$$
\int_{E} H[t(p)] D(p, T) d \nu=\int_{M^{*}} H\left(p^{*}\right) N\left(p^{*}, T, E\right) d \nu^{*},
$$

whenever either of the two integrals involved exists.

\section{Chapter IV. Applications to the plane}

4.1. We now wish to show that the theory developed in the previous chapters applies to the results of Radó and Reichelderfer [4] in the plane as outlined in the introduction. $M$ will be taken as a bounded, closed, oriented square in the $u v$-plane; $\nu(E)$ will be taken as the Lebesgue exterior measure of a set $E$ and will be denoted by $|E|$.

TheOREM. Let $T: p^{*}=t(w), w \in A \subset M, p^{*} \in A^{*} \subset M^{*}$ be a single-valued, continuous transformation from an analytic set $A$ in a bounded, closed, oriented square $M$ in the w-plane onto a set $A^{*} \subset M^{*} . A$ necessary and sufficient condition that $T$ be B.V. on $A$ (see $\$ 3.17$ ) is that there exist a finite constant $L$ such that $\nu^{*}\left[T\left(s_{1} A\right)\right]+\cdots+\nu^{*}\left[T\left(s_{k} A\right)\right]<L$ for every finite sequence of nonoverlapping, closed, oriented squares, $s_{1}, \cdots, s_{k}$ such that $s_{i} \subset M, i=1,2, \cdots, k$.

Proof. Assume that $T$ is B.V. on $A$. Let $s_{1}, \cdots, s_{k}$ be a finite sequence of nonoverlapping, closed, oriented squares in $M$. No point $p$ can be in more than four of the squares $s_{i}$. Hence $4 N\left[p^{*}, T,\left(s_{1}+\cdots+s_{k}\right) A\right] \geqq N\left(p^{*}, T, s_{1} A\right)$ $+\cdots+N\left(p^{*}, T, s_{k} A\right)$. Since $N\left(p^{*}, T, s_{i} A\right) \geqq 1$ for $p^{*} \in T\left(s_{i} A\right)$, 


$$
\begin{aligned}
\sum_{i=1}^{k} \nu^{*}\left[T\left(s_{i} A\right)\right] & \leqq \sum_{i=1}^{k} \int_{M^{*}} N\left(p^{*}, T, s_{i} A\right) d \nu^{*} \leqq 4 \int_{M^{*}} N\left[p^{*}, T,\left(s_{1}+\cdots+s_{k}\right) A\right] d \nu^{*} \\
& \leqq 4 \int_{M^{*}} N\left(p^{*}, T, A\right) d \nu^{*} .
\end{aligned}
$$

Since $T$ is B.V. on $A, N\left(p^{*}, T, A\right)$ is a $\nu^{*}$-summable function on $M^{*}$ and $L$ can be taken as any number greater than four times the definite integral of $N\left(p^{*}, T, A\right)$ over $M^{*}$.

Assume that there exists a finite constant $L$ such that $\nu^{*}\left[T\left(s_{1} A\right)\right]+\cdots$ $+\nu^{*}\left[T\left(s_{k} A\right)\right]<L$ for every finite system of nonoverlapping, closed, oriented squares $s_{1}, \cdots, s_{k}$ such that $s_{i} \subset M$. Let $D_{n}$ denote a subdivision of $M$ into $4^{n}$ congruent closed squares by lines parallel to the coordinate axes. Let $c\left(p^{*}, E^{*}\right)$ denote the characteristic function of the set $E^{*}$, that is, $c\left(p^{*}, E^{*}\right)=1$ if $p^{*} \in E^{*}$ and $=0$ if $p^{*} \in E^{*}$. Then

$$
g_{n}\left(p^{*}\right)=\sum c\left[p^{*}, T(s A)\right]
$$

where the summation is taken with respect to the squares $s$ in the subdivision $D_{n}$, is a $\nu^{*}$-measurable function since $c\left[p^{*}, T(s A)\right]$ is a $\nu^{*}$-measurable function for each $s$ in the subdivision $D_{n}$. Then (see Saks [5, Theorem 12.3, p. 27])

$$
\int_{M^{*}} g_{n}\left(p^{*}\right) d \nu^{*}=\sum \int_{M^{*}} c\left[p^{*}, T(s A)\right] d \nu^{*}=\sum \nu^{*}[T(s A)]<I
$$

where the summations are taken with respect to the squares $s$ in $D_{n}$. Since $g_{n}\left(p^{*}\right)$ is a nondecreasing sequence of non-negative, $\nu^{*}$-measurable functions, we have (see Saks [5, Theorem 12.6, p. 28]) $g\left(p^{*}\right)=\lim _{n \rightarrow \infty} g_{n}\left(p^{*}\right)$ is a $\nu^{*}$-measurable function and

$$
\int_{M^{*}} g\left(p^{*}\right) d \nu^{*}=\lim _{n \rightarrow \infty} \int_{M^{*}} g_{n}\left(p^{*}\right) d \nu^{*} \leqq L .
$$

Let $p^{*}$ be any point in $T(A)$ and let $k$ be any integer less than or equal to $N\left(p^{*}, T, A\right)$. Then there are $k$ distinct points $w_{1}, \cdots, w_{k}$ in $A$ such that $t\left(w_{i}\right)=p^{*}, i=1, \cdots, k$. For $n_{0}$ large enough no two of these points will lie in the same square of a subdivision $D_{n}$ for $n>n_{0}$. Hence $g_{n}\left(p^{*}\right) \geqq k$ for $n>n_{0}$ and thus $g\left(p^{*}\right) \geqq k$. Since this is true for every integer $k \leqq N\left(p^{*}, T, A\right)$, $g\left(p^{*}\right) \geqq N\left(p^{*}, T, A\right)$. Since $g\left(p^{*}\right)$ is a $\nu^{*}$-summable function, it follows that $N\left(p^{*}, T, A\right)$ is a $\nu^{*}$-summable function.

4.2. Theorem. Let $T: p^{*}=t(w), w \in A \subset M, p^{*} \in A^{*} \subset M^{*}$ be a single-valued, continuous transformation from an analytic set $A$ in a bounded, closed, oriented square $M$ in the w-plane onto a set $A^{*} \subset M^{*}$. If for every $\epsilon>0$ there exists an $\eta=\eta(\epsilon)>0$ such that $\nu^{*}\left[T\left(s_{1} A\right)\right]+\cdots+\nu^{*}\left[T\left(s_{k} A\right)\right]<\epsilon$ for every finite sequence of nonoverlapping closed, oriented squares $s_{1}, \cdots, s_{k}$ such that $s_{i} \subset M$ 
and $\left|s_{1}\right|+\cdots+\left|s_{k}\right|<\eta$, then $T$ is B.V. and A.C. on $A$ (see $\S 3.17,3.27$ ).

Proof. We first show that $T$ is B.V. on $A$. Let $n_{0}$ be an integer such that $|M| / 4^{n_{0}}<\eta(1)$. For the subdivision $D_{n}$ defined in the proof of the theorem in $\S 4.1$, we have $\nu^{*}\left[T\left(s_{0} A\right)\right]<1$ for each square $s_{0}$ in $D_{n_{0}}$. Hence, for any $n>n_{0}$, the summation $\nu^{*}[T(s A)]$ over the squares $s$ of $D_{n}$ which lie in a given square $s_{0}$ of $D_{n_{0}}$ is less than 1. Thus, for the functions $g_{n}\left(p^{*}\right), n>n_{0}$, defined in the proof of the theorem in $\$ 4.1$,

$$
\int_{M} g_{n}\left(p^{*}\right) d \nu^{*}=\sum \nu^{*}[T(s A)]<4^{n_{0}}
$$

where the summation is taken with respect to the squares $s$ in $D_{n}$. Hence $g\left(p^{*}\right)=\lim _{n \rightarrow \infty} g_{n}\left(p^{*}\right)$ is a $\nu^{*}$-summable function on $M^{*}$. Since $N\left(p^{*}, T, A\right)$ $\leqq g\left(p^{*}\right)$ on $A, N\left(p^{*}, T, A\right)$ is a $\nu^{*}$-summable function on $M^{*}$. Thus $T$ is B.V. on $A$.

We now show that $T$ is A.C. on $A$. Let $E$ be a set of Lebesgue measure zero in $M$. Let $\epsilon>0$ be given. We have an open set $O \supset E$ such that $|O|<\eta(\epsilon)$. As before, let $D_{n}$ denote the subdivision of $M$ into $4^{n}$ congruent squares by lines parallel to the coordinate axes. For each integer $n$, let $S_{n}$ be the squares $s$ of $D_{n}$ such that $s \subset O$ and $s$ is not in a square of $D_{n-1}$ which lies in $O$. Then, if $S$ is all the squares in $S_{1}, S_{2}, \ldots, S$ is a denumerable system of nonoverlapping squares $s_{1}, s_{2}, \cdots$ such that $O=s_{1}+s_{2}+\cdots$. Since $\left|s_{1}\right|+\cdots+\left|s_{n}\right|$ $\leqq|O|<\eta(\epsilon), \nu^{*}\left[T\left(s_{1} A\right)\right]+\cdots+\nu^{*}\left[T\left(s_{n} A\right)\right]<\epsilon$ for every integer $n$. Thus

$$
\nu^{*}[T(E A)] \leqq \nu^{*}[T(O A)] \leqq \nu^{*}\left[T\left(s_{1} A\right)\right]+\cdots+\nu^{*}\left[T\left(s_{n} A\right)\right]+\cdots \leqq \epsilon .
$$

Since $\epsilon>0$ is arbitrary, $\nu^{*}[T(E A)]=0$. Since $E$ was any set of Lebesgue measure zero in $M, T$ is A.C. on $A$.

REMARK. If $T$ is B.V. and A.C. on $A$, the converse of the preceding theorem follows from Theorem 13.2, Chapter I of Saks [5], since $\nu^{*}[T(s A)]$ $\leqq \mu(s, T)$.

4.3. Let $T$ be B.V. on $A$. Then (see $\$ 3.21$ )

$$
\mu(E, T)=\int_{E} D(w, T) d \nu+\mu_{s}(E, T), \quad E \in \mathcal{X}_{0}(T) .
$$

For each integer $n$ and set $E \in \mathcal{X}_{0}(T)$, let

$$
\mu_{n}(E, T)=\mu\left(E X_{n}, T\right)=\int_{E X_{n}} D(w, T) d \nu+\mu_{s}\left(E X_{n}, T\right) .
$$

We also set (see introduction) for each oriented, closed square $s \subset M$

$$
G(s)=\nu^{*}\left[T\left(s^{0} A\right)\right] .
$$

Given a point $w \in M$, let us consider a sequence of oriented, closed squares $s_{n}$ 
such that $w \in s_{n}^{0} \subset M$ and $\lim \left|s_{n}\right|=0$. If for every such sequence, the quotients $\mu\left(s_{n}^{0} A, T\right) /\left|s_{n}\right|$ converge to a finite limit (which is then necessarily the same for all such sequences), then this limit is called the derivative of $\mu(E, T)$ at $w$ and is denoted by $\mu^{\prime}(w, T) . \mu_{n}^{\prime}(w, T)$ and $G^{\prime}(w)$ are defined in the same way. We then have (see, for example, Saks [5, chap. IV, Theorem 7.3]) that $\mu^{\prime}(w, T)$ exists and is equal to $D(w, T)$ almost everywhere in $M$ and for each integer $n, \mu_{n}^{\prime}(w, T)$ exists and is equal to $D(w, T)$ almost everywhere in $X_{n}$.

Lemma. $G^{\prime}(w)$ exists and is equal to $D(w, T)$ almost everywhere in $M$.

Proof. Let $w$ be a point in $X_{n}$ where $\mu_{n}^{\prime}(w, T)$ and $\mu^{\prime}(w, T)$ exist and are equal to $D(w, T)$. Then for any square $s \subset M$ such that $w \in s^{0}$,

$$
\mu_{n}\left(s^{0} A, T\right) /|s|=\mu_{n}\left(s^{0} X_{n}, T\right) /|s| \leqq G(s) /|s| \leqq \mu\left(s^{0} A, T\right) /|s| .
$$

Thus $G^{\prime}(w)$ exists and is equal to $D(w, T)$. Hence $G^{\prime}(w)$ exists and is equal to $D(w, T)$ almost everywhere on $A-A_{\infty}$.

Since $\mu\left[\mathcal{C}\left(A-A_{\infty}\right), T\right]=0, D(w, T)=0$ almost everywhere on $\mathcal{C}\left(A-A_{\infty}\right)$. Let $w \in \mathcal{C}\left(A-A_{\infty}\right)$ be a point where $\mu^{\prime}(w, T)$ exists and is equal to $D(w, T)=0$. Then for any square $s \subset M$, w $\in s^{0}$, by $\S 3.18, G(s) \leqq \mu\left(s^{0} A, T\right)$.

Hence, $G^{\prime}(w)=0$.

Therefore, $G^{\prime}(w)$ exists and is equal to $D(w, T)$ almost everywhere in $M$.

4.4. It is easily shown that the conditions B.V.B and A.C.B as given by Radó and Reichelderfer in terms of $G(s)=\nu^{*}\left[T\left(s^{0} B\right)\right]$ are equivalent to the conditions stated in the theorems in $\$ 4.1$ and 4.2 respectively in terms of $\nu^{*}[T(s B)]$ (note, one uses $s^{0}$ and the other $s$ ). If $M^{*}$ is taken as a closed, oriented square in the $x y$-plane and $\nu^{*}\left(E^{*}\right)$ is taken as the Lebesgue exterior measure of a set $E^{*} \subset M^{*}$, then, from $\S \S 4.1,4.2,4.3$, our theory on metric foundations of continuous transformations applies to the results of Radó and Reichelderfer [4] as given in the introduction.

\section{BIBLIOGRAPHY}

1. H. Hahn, Reelle Funktionen, vol. 1, Leipzig, 1932.

2. F. Hausdorff, Mengenlehre, 3d ed., Berlin, 1935.

3. C. Kuratowski, Topologie, I, Warsaw-Lwow, 1933.

4. T. Rad6 and P. Reichelderfer, $A$ theory of absolutely continuous transformations in the plane, Trans. Amer. Math. Soc. vol. 49 (1941) pp. 258-307.

5. S. Saks, Theory of the integral, New York, Stechert, 1937.

The Ohio State University, Columbus, Оhio. 